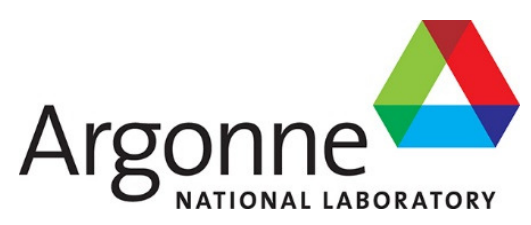

\title{
Micro Reactor Simulation Using the PROTEUS Suite in FY19
}

Nuclear Science and Engineering Division 


\begin{abstract}
About Argonne National Laboratory
Argonne is a U.S. Department of Energy laboratory managed by UChicago Argonne, LLC under contract DE-AC02-06CH11357. The Laboratory's main facility is outside Chicago, at 9700 South Cass Avenue, Argonne, Illinois 60439. For information about Argonne and its pioneering science and technology programs, see www.anl.gov.
\end{abstract}

\title{
DOCUMENT AVAILABILITY
}

Online Access: U.S. Department of Energy (DOE) reports produced after 1991 and a growing number of pre-1991 documents are available free via DOE's SciTech Connect (http://www.osti.gov/scitech/)

\author{
Reports not in digital format may be purchased by the public from the \\ National Technical Information Service (NTIS): \\ U.S. Department of Commerce \\ National Technical Information Service \\ 5301 Shawnee Rd \\ Alexandra, VA 22312 \\ www.ntis.gov \\ Phone: (800) 553-NTIS (6847) or (703) 605-6000 \\ Fax: (703) 605-6900 \\ Email: orders@ntis.gov \\ Reports not in digital format are available to DOE and DOE contractors from the \\ Office of Scientific and Technical Information (OSTI): \\ U.S. Department of Energy \\ Office of Scientific and Technical Information \\ P.O. Box 62 \\ Oak Ridge, TN 37831-0062 \\ www.osti.gov \\ Phone: (865) 576-8401 \\ Fax: (865) 576-5728
}

\section{Disclaimer}

This report was prepared as an account of work sponsored by an agency of the United States Government. Neither the United States Government nor any agency thereof, nor UChicago Argonne, LLC, nor any of their employees or officers, makes any warranty, express or implied, or assumes any legal liability or responsibility for the accuracy, completeness, or usefulness of any information, apparatus, product, or process disclosed, or represents that its use would not infringe privately owned rights. Reference herein to any specific commercial product, process, or service by trade name, trademark, manufacturer, or otherwise, does not necessarily constitute or imply its endorsement, recommendation, or favoring by the United States Government or any agency thereof. The views and opinions of document authors expressed herein do not necessarily state or reflect those of the United States Government or any agency thereof, Argonne National Laboratory, or UChicago Argonne, LLC. 
ANL/NSE-19/33

\section{Micro Reactor Simulation Using the PROTEUS Suite in FY19}

prepared by

Changho Lee, Yeon Sang Jung, and Hyoung Kyu Cho

Nuclear Science and Engineering Division, Argonne National Laboratory

September 30, 2019 



\section{ABSTRACT}

Two micro reactor cores were simulated using the MOC solver of PROTEUS: MegaPower for a heat pipe micro reactor and Holos-Quad for a high-temperature gas-cooled micro reactor. Verification of PROTEUS standalone was performed by simulating 2D and 3D core benchmark problems developed based on MegaPower and Holos-Quad. Simulation results indicated that PROTEUS successfully modelled 2D and 3D cores of the MegaPower and Holos-Quad micro reactors and PROTEUS eigenvalues agreed well with the SERPENT Monte Carlo solutions.

For a heat pipe cooled micro reactor, multiphysics simulations were performed using PROTEUS / FLUENT / ANLHTP. The coupling system of the three codes were developed using Python-based drivers, which exchange the data (power, temperature, and heat rate) required for the coupling and control the boundary conditions of FLUENT and ANLHTP. The coupled system was qualitatively verified using a 3D unit assembly problem composed of six fuel rods and seven heat pipes, which was developed based on the MegaPower compositions. The steady-state problem was solved using FLUENT / ANLHTP with given asymmetric fuel powers, demonstrating the reasonable convergence of temperatures of 7 heat pipes. Transient simulations were also performed by making one out of 7 heat pipes fail. For the transient, the coupled system of PROTEUS / FLUENT / ANLHTP demonstrated reasonable changes of total power and heat pipe temperatures with time, accounting for temperature feedback effects. In this simulation, no effect of structural change was accounted for, which will be performed using ANSYS-mechanical in future along with further verification with larger or whole-core 3D problems. 


\section{TABLE OF CONTENTS}

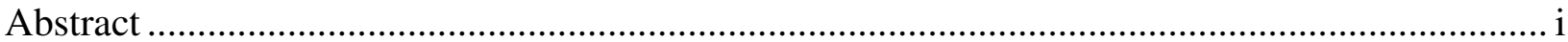

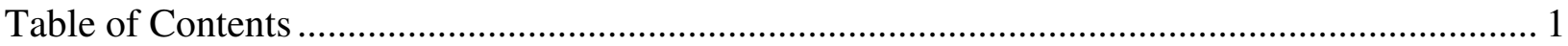

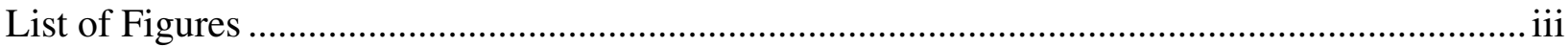

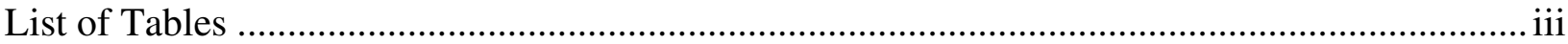

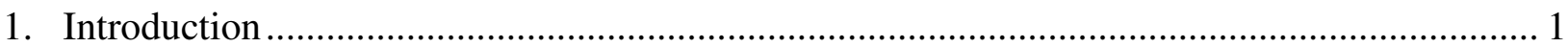

2. Computer Codes Used in Micro Reactor Simulation............................................................ 3

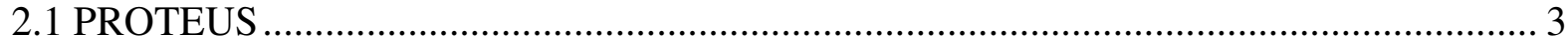

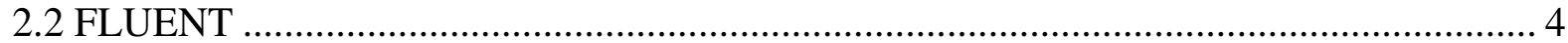

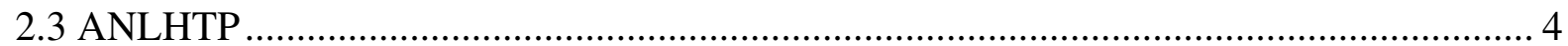

3. Neutronics Simulation of Micro Reactor Using PROTEUS ............................................ 10

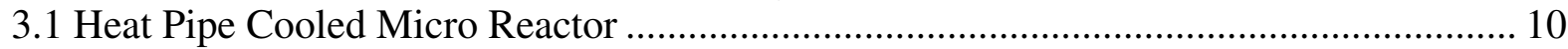

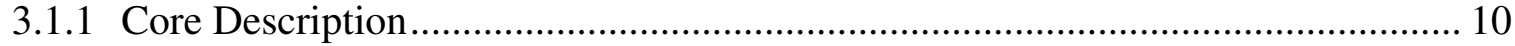

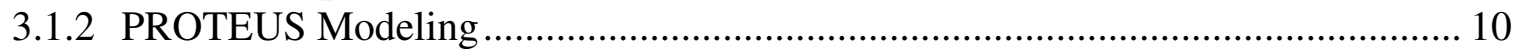

3.1.3 PROTEUS Solutions ................................................................................... 13

3.2 High-Temperature Gas-cooled Micro Reactor......................................................... 17

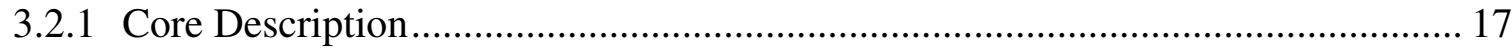

3.2.2 PROTEUS Geometry and Mesh....................................................................... 18

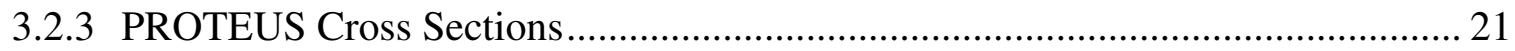

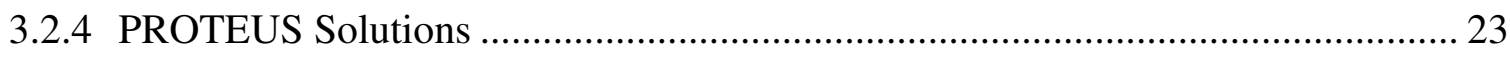

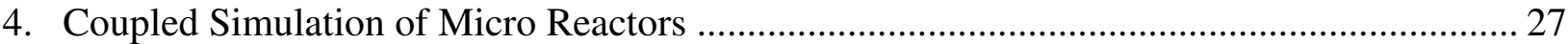

4.1 Coupled System of PROTEUS / FLUENT / ANLHTP ............................................. 27

4.2 Coupled Calculation Results ...................................................................................... 30

4.2.1 Steady-state Single Heat Pipe Problem .......................................................... 30

4.2.2 Steady-state Multi Heat Pipe Problem............................................................... 31

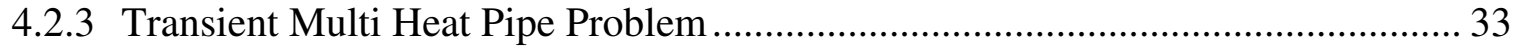

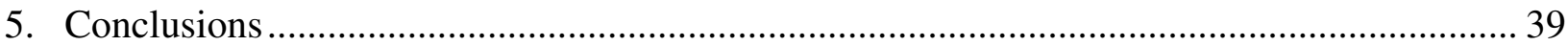

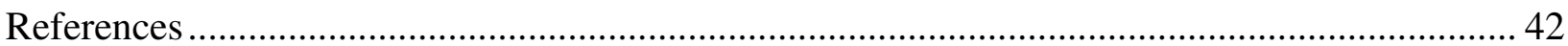




\section{LIST OF FIGURES}

Figure 2-1. Overview of the PROTEUS System ............................................................. 3

Figure 2-2. ANLHTP Calculation Flow ….............................................................. 7

Figure 2-3. ANLHTP Thermal Resistance Network ..................................................... 8

Figure 2-4. ANLHTP Validation Results ........................................................................ 8

Figure 2-5. Modification of ANLHTP for Smooth Transition of Heat Transport with

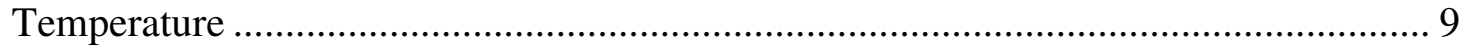

Figure 3-1. Neutron Flux Spectra of MegaPower Fuel Assembly and Core ........................ 11

Figure 3-2. Geometry and Mesh Generation for the MegaPower Reactor ............................ 12

Figure 3-3. Benchmark Problems with Different Control Material Locations in the Control

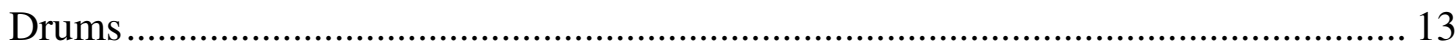

Figure 3-4. Comparison of Axial Power Profiles between SERPENT and PROTEUS for 3D

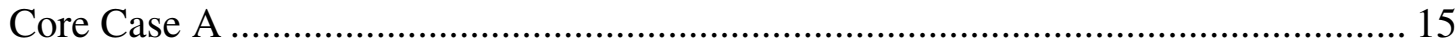

Figure 4-5. Geometry and Mesh Scheme of Single Heat Pipe Problem ............................... 31

Figure 4-6. Temperature Distribution for Single Heat Pipe Problem .................................... 31

Figure 4-7. Geometry and Mesh Scheme of Multi-Heat Pipe Problem................................ 32

Figure 4-8. Temperature Distribution of Multi-Heat Pipe Problem ..................................... 33

Figure 4-9. Heat Pipe Wall Temperature Convergence Result of Multi-Heat Pipe Problem .. 33

Figure 4-10. Temperature Distributions of the HP1 Failure Problem Before and After

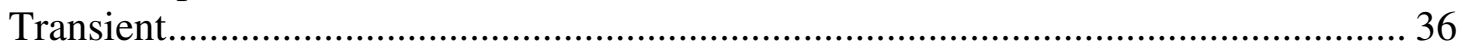

Figure 4-11. Wick-vapor Interface Temperature Transient for the HP1 Failure Problem....... 36

Figure 4-12. Temperature Distributions of the HP2 Failure Problem Before and After

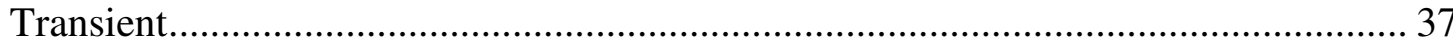

Figure 4-13. Wick-vapor Interface Temperature Transient for the HP2 Failure Problem....... 37

Figure 4-14. Relative Power (Top) and Heat Pipe Temperature (Bottom) Change with Time

for One Heat Pipe Failure Transient Problem........................................................... 38

\section{LIST OF TABLES}

Table 3-1. Key Specifications of the MegaPower Reactor [1,2] ........................................ 10

Table 3-2. Eigenvalues of MegaPower Test Cases from SERPENT .................................... 14

Table 3-3. Eigenvalue and Control Worth Comparison between SERPENT and PROTEUS for

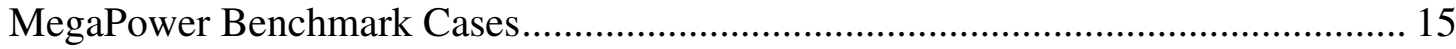

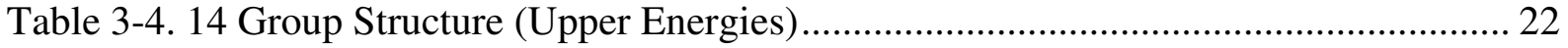

Table 3-6. Benchmark Results for 3D Holos Core Problems ................................................ 24

Table 4-1. Fuel Rod Powers for FLUENT / ANLHTP with the Steady-state Condition ........ 32 



\section{Introduction}

In recent years, concerns in compact power generation are reemerging due to the growing demand of affordable and sustainable energy resources even in remote locations, military bases, etc. where electricity supply is limited. Since nuclear energy would be a good energy source for such purpose, efforts have been made to develop micro reactors such as MegaPower [1,2], eVinci [3], and Holos-Quad [4] which are small nuclear reactors generating power ranging from a few $\mathrm{kWe}$ to a few tens MWe. The desirable key characteristics of micro reactors are sustainability, transportability, safety, controllability, easy installation, maintenance, etc., in order to produce robust, self-contained, and long-term power. Since most micro reactors are compact and of irregular geometry, Monte Carlo codes are often used to simulate them. However, due to the current limitations of Monte Carlo approaches in performing transient, thermal expansion analyses, etc., deterministic codes would be better to use if they are able to solve such reactor problems with irregular geometry.

For deterministic neutronics analysis of micro reactors, we used PROTEUS which is a highfidelity capable three-dimensional (3D) deterministic neutron transport code developed by Argonne National Laboratory (ANL) under the Department of Energy (DOE) Nuclear Energy Advanced Modeling and Simulation (NEAMS) program. Being able to solve the neutron transport equation in steady-state and transient conditions, the code contains three solvers for flexible applications: the second-order discrete ordinates (SN) [5] and method of characteristics (MOC) [6] transport solvers based on unstructured finite element meshes as well as the nodal transport solver (NODAL) [7] for hexagonal and Cartesian geometries for use in rapid design application. The SN and MOC solvers of PROTEUS can be used for simulating micro reactors which are mostly of irregular geometry. The SN solver is preferred to use for fast reactor systems where the heterogeneity effect is not severe, while the MOC solver is efficient to analyze thermal systems where neutron fluxes largely change over heterogeneous regions. A thermal expansion behavior, which is an important and challenging phenomena of metal-fueled fast reactors, can be simulated by the unstructured mesh based solvers of PROTEUS.

For preliminary verification efforts of neutronics solutions, we simulated two types of micro reactors using PROTEUS: MegaPower for a heat pipe cooled micro reactor and the Holos-Quad for a high temperature gas cooled micro reactor. Since the geometries of the cores are nonstandard, CUBIT [8] was primarily used to generate geometry and mesh for the cores, and the Argonne mesh toolkit [9] was used as well to support CUBIT generated meshes. Multigroup cross sections for each core were generated using the SERPENT [10] / GenISOTXS [11] process. Since MegaPower is a fast-spectrum core, we tried cross sections generated from $\mathrm{MC}^{2}-3$ [12] as well because Monte Carlo generated cross sections have accuracy issues in high-order scattering moments. PROTEUS solutions for different core configurations of two-dimensional (2D) and 3D cores of each micro reactor were compared with SERPENT solutions.

An initial effort of deterministic multiphysics simulation for a heat pipe cooled micro reactor was made using PROTEUS for neutronics analysis, ANSYS [13] for thermal mechanical analysis, and ANLHTP [14] for heat pipe performance analysis. For analyzing a heat pipe cooled

micro reactor, FLUENT [15] in the ANSYS package was used in the simulation here since the evaluation version of it was available. Multiphysics simulation was limited to considering heat 
transfer only, not accounting for thermal expansion. However, the same coupling mechanism used for the coupled system of PROTEUS / FLUENT / ANLHTP can be easily applied to ANSYS-mechanical with minor efforts.

The ANLHTP code is a one-dimensional heat pipe performance code that developed by ANL in the 1980s. The code was resurrected in the work, verified and validated again against a few heat pipe experiments. During the $\mathrm{V} \& \mathrm{~V}$ process several updates were made in terms of solution accuracy and convergence as well.

For the coupled system of PROTEUS / FLUENT / ANLHTP, Python scripts were developed to transfer data between the three codes. Since a geometry and mesh file cannot be shared between PROTEUS and FLUENT at the moment, we used different mesh files from each other which required the interpolation of data to exchange between the two codes.

The coupled codes were tested to simulate a small fuel assembly problem composed of 6 fuel rods and 7 heat pipes with $180 \mathrm{~cm}$ high. A steady-state problem of the core was solved first and extended to a transient problem with the failure of one out of 7 heat pipes.

Sections 2 introduces the codes used in modeling and simulation of micro reactors, and Section 3 presents neutronic simulation using PROTEUS for two micro reactors (MegaPower and Holos-Quad). Section 4 demonstrates the use of the coupled system of PROTEUS / FLUENT / ANLHTP for simulation of benchmark problems for a heat pipe cooled reactor. Conclusions are discussed in Section 5. 


\section{Computer Codes Used in Micro Reactor Simulation}

\subsection{PROTEUS}

The PROTEUS code is a high-fidelity capable neutron transport code based on finite element discretization of the domain, which has been developed under the DOE-NE NEAMS program. The $S_{N}$ and MOC transport solvers are available in PROTEUS to solve heterogeneous geometry problems with no or minimal geometrical approximations. The nodal transport solver based on homogenized assemblies and structured geometry was also implemented in PROTEUS to provide a conventional-fidelity level of solutions in a consistent framework for use in rapid design application. In the NODAL solver, two methodology options are available: $\mathrm{P}_{\mathrm{N}}$ and Simplified $\mathrm{P}_{\mathrm{N}}$ $\left(\mathrm{SP}_{\mathrm{N}}\right)$. The $\mathrm{P}_{\mathrm{N}}$ approach is the identical methodology used in DIF3D-VARIANT [16] although the release version only handles diffusion theory on Cartesian, hexagonal, and triangular-z grids. For the $\mathrm{SP}_{\mathrm{N}}$ approach [17], a transverse integrated nodal methodology was built on the hexagonal grid model utilizing up to a $\mathrm{SP}_{3}$ approximation.

All the three solvers are able to solve steady-state and transient problems with the built-in thermal fluid calculation capability. The gamma transport calculation is possible to accurately solve for power distributions. In the NODAL solver, the molten salt reactor (MSR) capability was implemented along with relevant thermal fluid modules so that it is able to solve MSR problems, accounting for redistribution of delayed neutron precursor concentrations due to fuel flow velocities inside and outside the core.

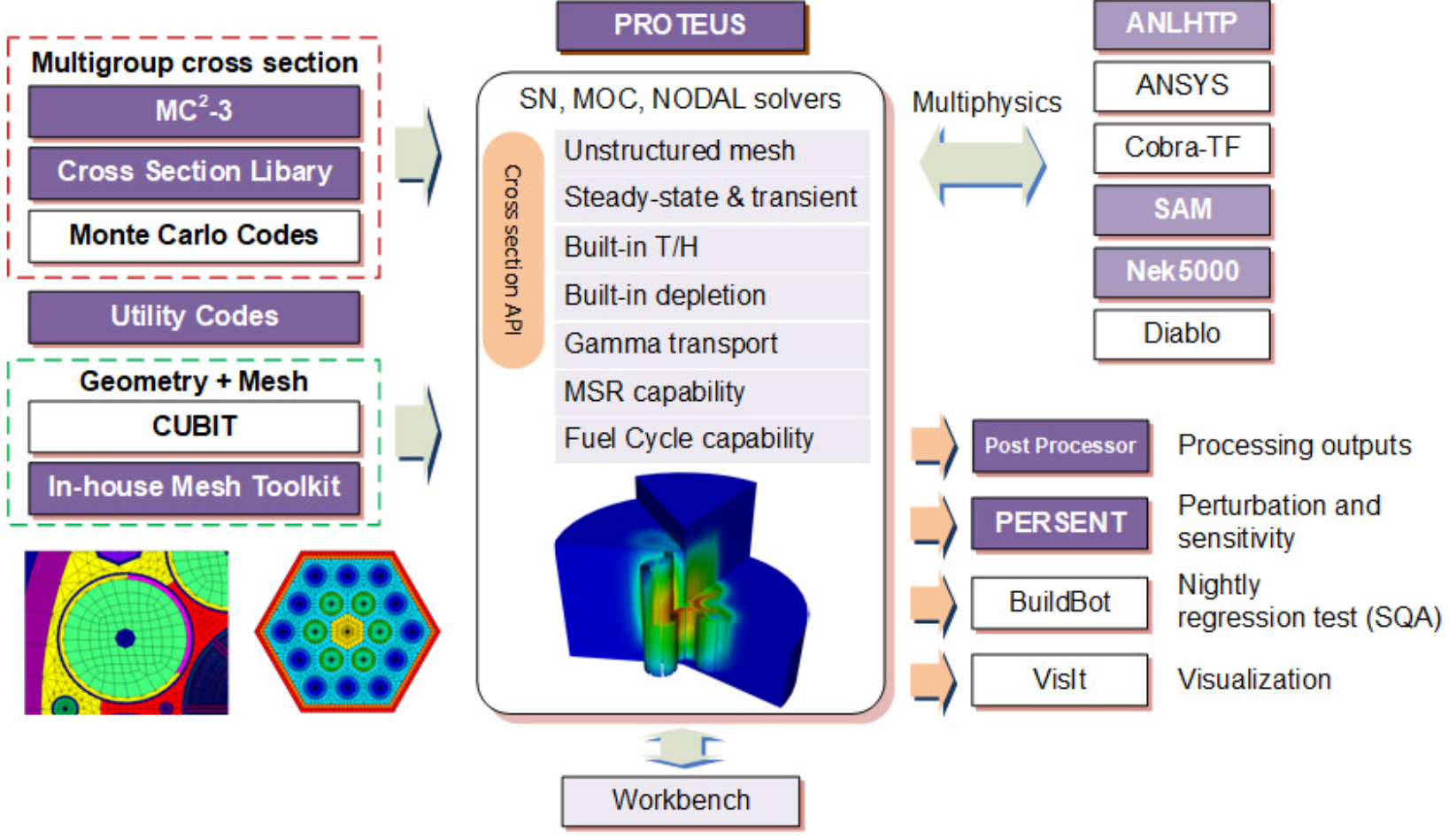

(The purple-colored boxes denote the codes developed by Argonne)

Figure 2-1. Overview of the PROTEUS System 
The fuel cycle capability as well as depletion was recently implemented in the NODAL solver to meet needs for actual reactor core design and analysis. The NODAL solver is able to generate the CCCC interface files in order to run PERSENT for reactivity perturbation and sensitivity analysis.

Cross sections for PROTEUS can be generated in the ISOTXS format using $\mathrm{MC}^{2}-3$ and Monte Carlo codes (Serpent and OpenMC). With the Monte Carlo codes, the GenISOTXS code produces ISOTXS files which can be combined to produce the ISOPAR format with state parameters in terms of temperature, burnup, control rod, void fraction, etc.

Geometry and mesh are generated using CUBIT standalone or a combination of CUBIT and the Argonne mesh toolkit. For conventional hexagonal and Cartesian geometry problems, the Argonne mesh toolkit is able to easily generate meshes with text-type keyword inputs. Recently, the NEAMS Workbench has been updated with PyPROTEUS which help users create PROTEUS inputs, run the code, and post-process code outputs.

Multiphysics simulations with other physics tools such as Nek5000, Diablo, Cobra-TF, SAM, ANSYS, and ANLHTP. Currently, Nek5000 and Diablo work with PROTEUS-SN, Cobra-TF and ANSYS can be coupled with PROTEUS-MOC, and SAM (a MOOSE-based code) works with PROTEUS-NODAL. The coupling approach that we employed for the coupling of SAM and PROTEUS-NODAL will be extended for coupling with other MOOSE-based codes in the future.

Figure 2-1 shows the overview of the PROTEUS suite, including PROTEUS capabilities and the codes that were developed by ANL (purple-colored boxes) or used to support PROTEUS and multiphysics simulation.

\subsection{FLUENT}

FLUENT is a well-known computer program for modeling fluid flow, heat transfer, and chemical reactions in complex geometries. The code provides complete mesh flexibility, including the ability to solve your flow problems using unstructured meshes that can be generated about complex geometries with relative ease. The code allows users to simulate: 1) 2D planar, 2D axisymmetric, 2D axisymmetric with swirl (rotationally symmetric), and 3D flows, 2) Flows on quadrilateral, triangular, hexahedral (brick), tetrahedral, wedge, pyramid, polyhedral, and mixed element meshes, 3) Steady-state or transient flows, 4) Incompressible or compressible flows, including all speed regimes (low subsonic, transonic, supersonic, and hypersonic flows), 5) Inviscid, laminar, and turbulent flows, 6) Newtonian or non-Newtonian flows, 7) Heat transfer, including forced, natural, and mixed convection, conjugate (solid/fluid) heat transfer, and radiation, and so on. FLUENT is ideally suited for incompressible and compressible fluid-flow simulations in complex geometries.

\subsection{ANLHTP}

For thermal analysis of a heat pipe cooled reactor core, a heat pipe code is required, which can evaluate the amount of the heat removed by heat pipes for given conditions. In this study, we selected ANLHTP, which is a one-dimensional heat pipe analysis code developed at ANL in the 1980s. The code was developed to simulate a sodium heat pipe based on theory, analyses, and experimental data presented by Chi [18] and Dunn and Reay [19]. For simplification, it was assumed that the evaporator and condenser are nearly isothermal (at uniform temperature) and 
there is negligible axial heat conduction along the pipe wall or wick. These assumptions allow the code to calculate the heat transfer rate without solving differential equations of fluid and solid structures.

The input data of ANLHTP are pipe geometry, working fluid, thermal boundary conditions, operation mode, etc. Based on the input data, the code calculates wick parameters (being able to handle covered groove, open groove, screen, and screen \& artery wick types) and makes an initial guess for the heat transfer rate to solve the non-linear equation. Then, the flow rates in each part of the heat pipe and the required thermal resistances are evaluated. With these, the heat transfer rate is updated. Iterations are made until the convergence on the heat transfer is achieved. Then, the code compares the estimated value with five operational limits of a heat pipe including viscous, sonic, entrainment, boiling and capillary limits.

The simplified calculation procedure of the code is plotted in Figure 2-2 and the key numerical procedure of ANLHTP is denoted with a blue line. In this procedure, the code calculates the thermal resistances at each part of the heat pipe as illustrated in Figure 2-3. Among them, resistances 5-7 are for the temperature changes along the vapor path. These resistances are small in most cases but cannot be neglected if the vapor pressure is low so that the Mach number in the vapor core is large or the compressibility effects are significant. The resistances in the vapor core are calculated using the temperature drops evaluated from the pressure drops. The pressure drops are calculated using the one-dimensional momentum conservation equation which requires constitutive equations for the friction factors depending on the flow condition. The code incorporates the friction factor models for both incompressible and compressible flows, either of which is applied considering the Mach number criterion, 0.2 , for the compressibility. A reduced form of the one-dimensional equation is applied, enabling the code to estimate the pressure drops including the compressibility effect in the adiabatic region and the pressure recovery in the condenser. For the thermal resistances 1-3 and 9-11, the analytical thermal resistance model for a circular tube is applied. For the wick region, the effective thermal conductivity of the saturated wick is used along with the wick's radii. The thermal resistances at the boiling and condensing surfaces are calculated using the empirical model presented by Dunn and Reay [19].

For the capillary limit calculation, ANLHTP uses the pressure drop calculation results for both liquid and vapor. It compares the total pressure drop occurring at the beginning of the evaporator and the maximum capillary pressure difference for the limiting case when the meniscus is flat and a wet point exists. The total pressure drop should be smaller than the maximum capillary pressure difference to sustain the circulation. Otherwise, the heat pipe is assumed to reach its operational limit. At the same time, it checks if the liquid pressure exceeds the vapor pressure at any axial location. If the pressures of the liquid are all less than the corresponding vapor pressure, the flow would be stable. Otherwise, the code assumes that the operation limit is reached. In this manner, the capillary limit of the heat pipe can be evaluated. For other operational limits, empirical correlations for the limiting criteria were implemented and details of the models are summarized in Reference [14].

ANLHTP has been validated against two existing experimental results with sodium heat pipes: one was the operation limit test result conducted at Los Alamos National Laboratory (LANL) [20] and the other ANL's Heat Pipe Test Facility (HPTF) [21]. For both experiments, ANLHTP showed reasonable prediction as shown in Figure 2-4.

In the LANL experiment, a 1 inch diameter and $1.1 \mathrm{~m}$ length sodium heat pipe was operated over a large range of evaporation inlet conditions, using the screen \& artery wick. The heat was supplied to the apparatus by induction coils and rejected though a gas calorimeter. In the 
simulation, the input temperatures were ranging from 600 to $875{ }^{\circ} \mathrm{C}$, and the sink temperatures were varied to search for performance limits. Figure 2-4(a) shows the comparison between the experimental data and the ANLHTP results for the operation limit. The ANLHTP results show very good agreement with the measurement. The code calculations indicated that the heat pipe performance met the sonic limitation below $630{ }^{\circ} \mathrm{C}$, while in the range of $630-650{ }^{\circ} \mathrm{C}$, the performance was limited by the capillary and pressure limitations. For the higher evaporator temperatures, the code indicated that the heat transport was limited by the entrainment limit.

In the HPTF experiment, a sodium heat pipe of 2 inch diameter and $0.7 \mathrm{~m}$ length was tested with various input powers to the evaporator. It consisted of an Incoloy 800 pipe, 2-1/2 wraps of 100 mesh screen lining inside wall, and two longitudinal arteries of 0.125 inch inner diameter. The steady-state heat pipe data were obtained under 19 power conditions, and the temperatures at the evaporator were measured. The heat transport rates and temperatures were in the range of 6.51-8.39 $\mathrm{kW}$ and $760-871^{\circ} \mathrm{C}$, respectively. These conditions were well below the operational limits of the heat pipe and in its operating states. In this context, the HPTF experiment was an operational performance test. As shown in Figure 2-4(b), the linearly increasing trend of the heat transport rate along with the evaporator temperature was well reproduced by ANLHTP.

The original ANLHTP was improved for coupling with a commercial thermal analysis code FLUENT. At first, its robustness was enhanced by removing the discontinuity or oscillation in physical models when a flow regime inside a heat pipe undergoes a laminar-turbulent or incompressible-compressible flow transition as shown in Figure 2-5. This feature accelerated the convergence characteristic of the Picard iteration for the coupled simulation between ANLHTP and FLUENT. Secondly, solid properties for new material were added for various wick structure modeling. Finally, input and output processes were modified to handle the code using a MATLAB or Python script which was written to control the boundary conditions of ANLHTP. This improved ANLHTP and the scripts were used for the coupled simulation.

As ANLHTP was developed to predict heat pipe performance and temperature distributions during a steady-state operation, its application is limited to the steady-state conditions or possibly slow transient conditions. Moreover, it assumes a uniform heat flux distribution in calculating the pressure drop, which may cause errors if the heat flux is not uniform significantly and the pressure drop in the vapor core is considerable with compressibility. Therefore, quantitative analyses for the errors caused by these assumptions are required and further improvements would be necessary in future. 


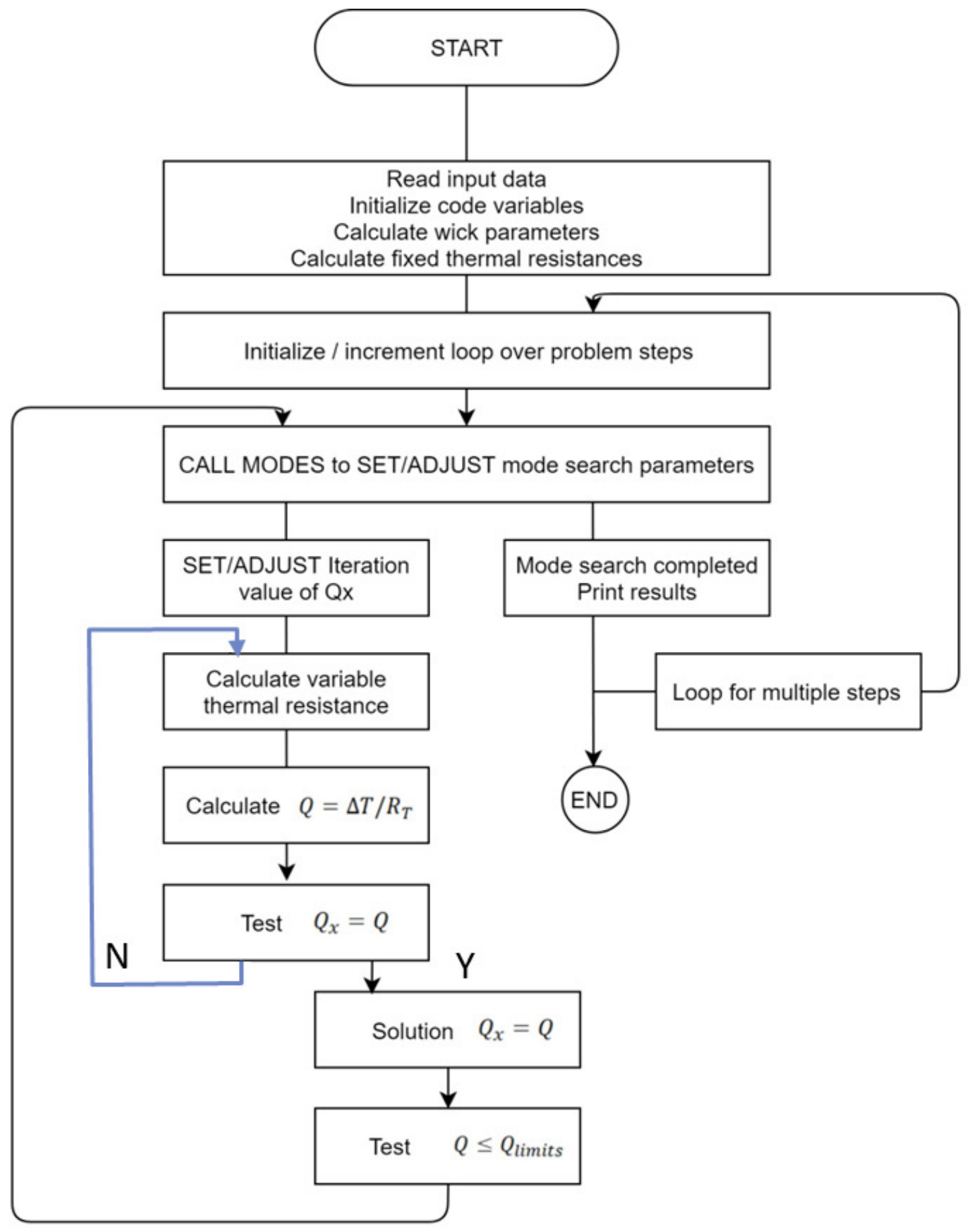

Figure 2-2. ANLHTP Calculation Flow 


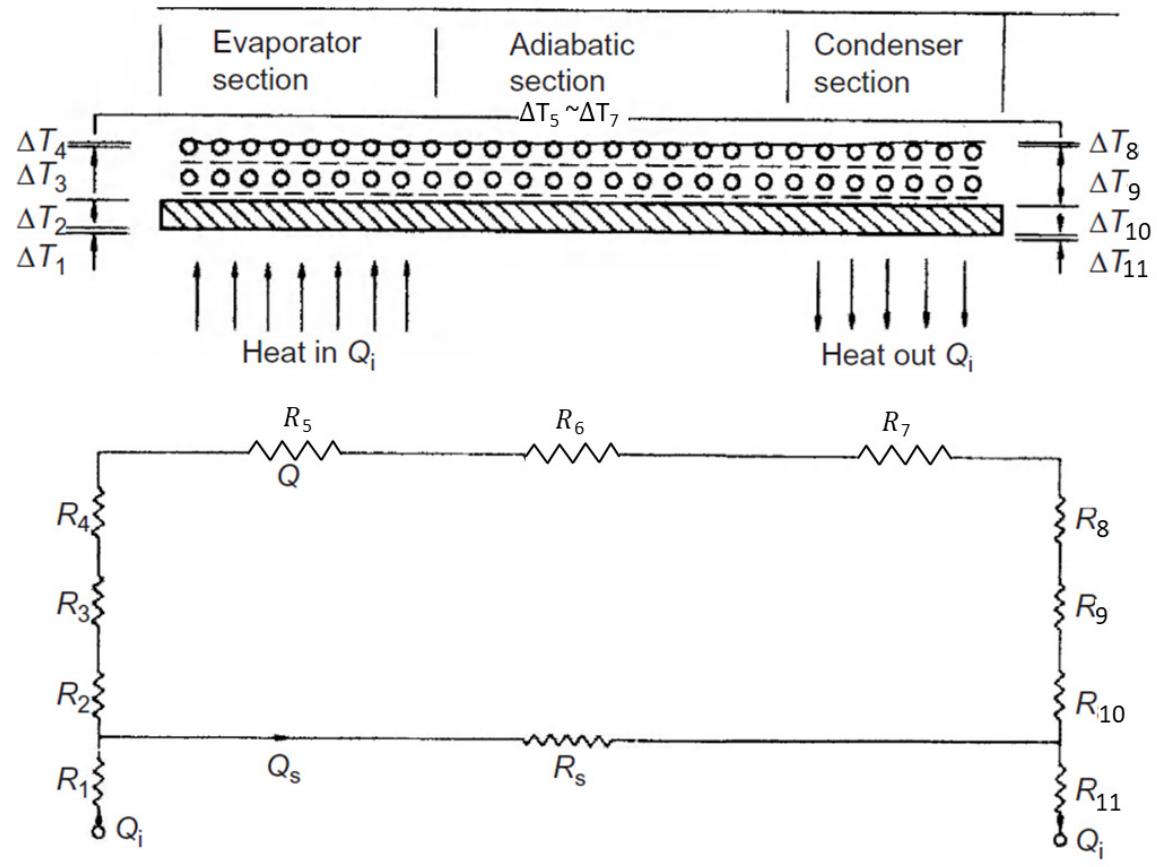

Figure 2-3. ANLHTP Thermal Resistance Network

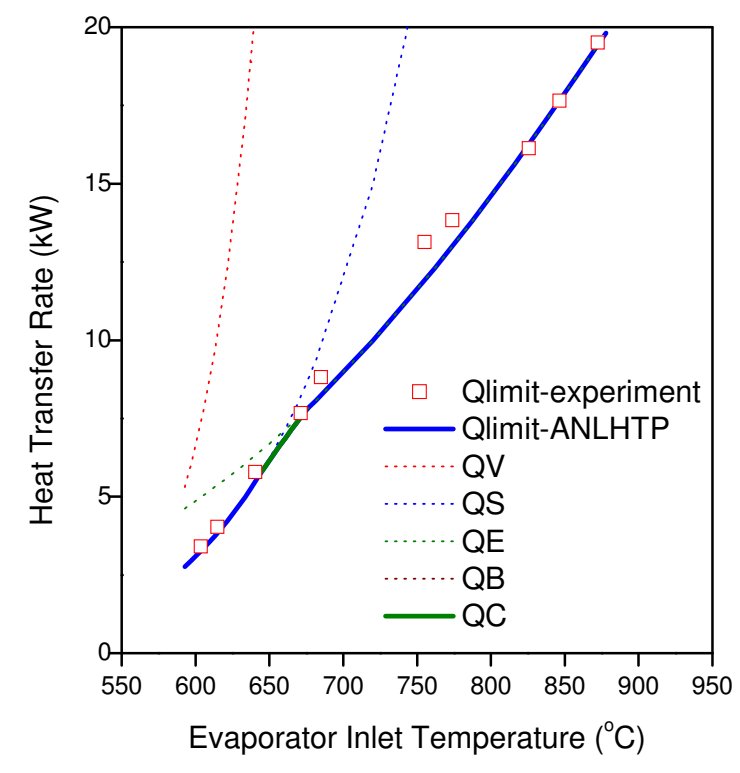

a) LANL Sodium Heat Pipe Test Simulation

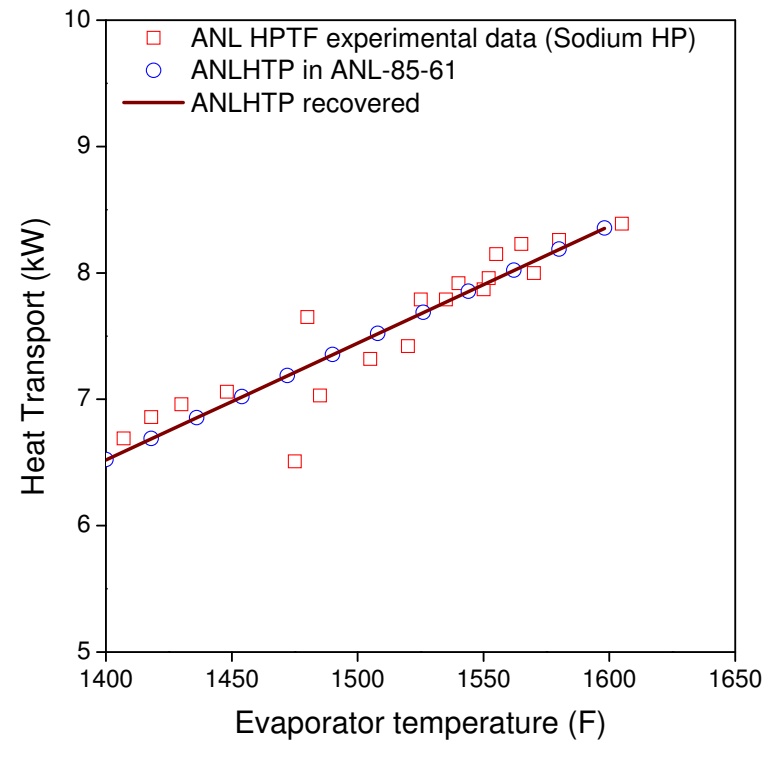

(b) ANL HPTF Test Simulation

Figure 2-4. ANLHTP Validation Results 

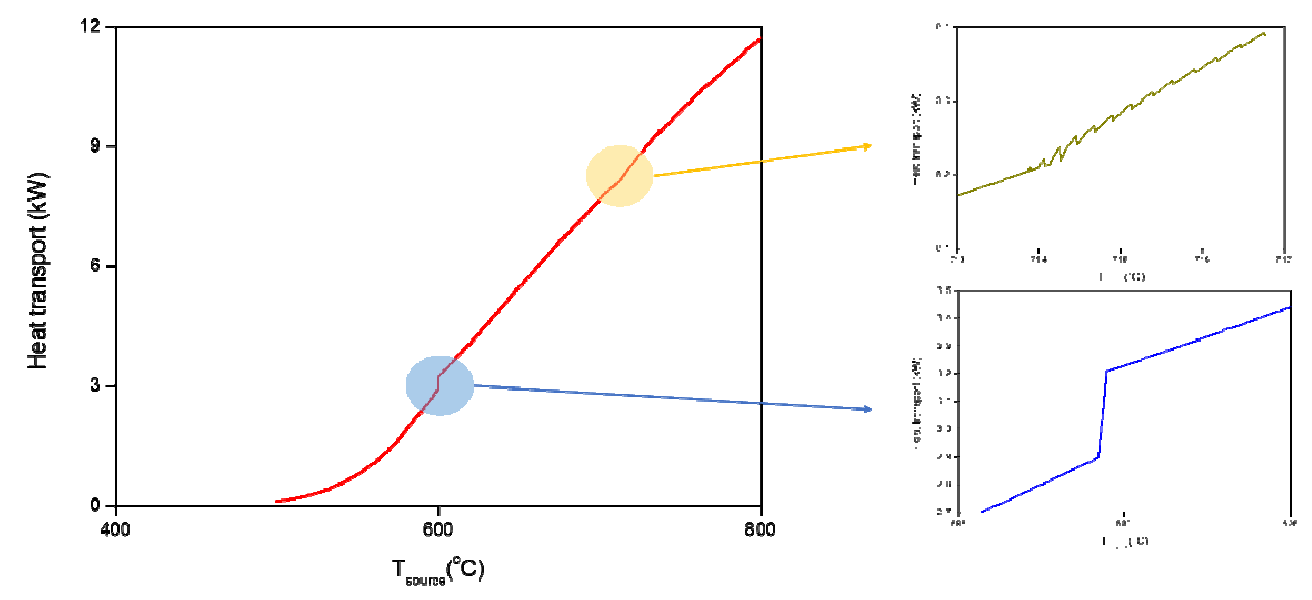

(a) Before modification
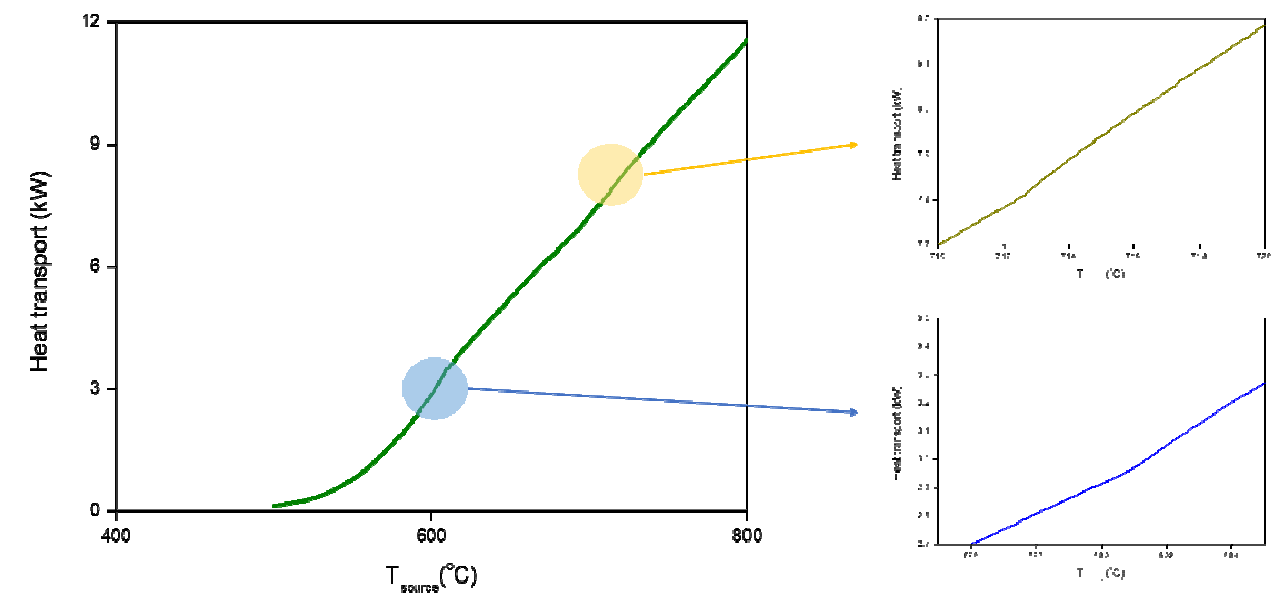

(b) After modification

Figure 2-5. Modification of ANLHTP for Smooth Transition of Heat Transport with Temperature 


\section{Neutronics Simulation of Micro Reactor Using PROTEUS}

\subsection{Heat Pipe Cooled Micro Reactor}

\subsubsection{Core Description}

The MegaPower reactor is a micro reactor based on a heat pipe cooled reactor concept, developed by the Los Alamos National Laboratory (LANL). Key characteristics of the reactor system are $5 \mathrm{MWth}(2 \mathrm{MWe})$, heat pipe cooled, $19.75 \mathrm{wt} \%$ enriched $\mathrm{UO}_{2}$ fuel, stainless steel monolith, and drum-type control system. The coolant material is liquid metal potassium which flows though heat pipes at $675^{\circ} \mathrm{C}$. This reactor uses an alumina $\left(\mathrm{Al}_{2} \mathrm{O}_{3}\right)$ reflector with 12 embedded control drums that contain an arc-type control material $\left(\mathrm{B}_{4} \mathrm{C}\right)$ for reactivity control. The height of the active core is $150 \mathrm{~cm}$ with top and bottom reflectors of $15 \mathrm{~cm}$ high each. Two emergency shutdown control rods - 1 solid rod and 1 annular tube - can be inserted to the central hexagonal area. The specifications of the MegaPower micro reactor are summarized in Table 3-1.

We chose the MegaPower reactor as a micro reactor benchmark model in this simulation study since all reactor specification data are available from the references $[1,2]$. Note that some minor or uncertain geometry details were simplified for benchmark models that were simulated since the objective of this study is to demonstrate modeling and simulation capabilities of PROTEUS for micro reactors.

Table 3-1. Key Specifications of the MegaPower Reactor [1,2]

\begin{tabular}{|c|c|c|c|}
\hline Parameter & Value & Parameter & Value \\
\hline \multicolumn{2}{|c|}{$\begin{array}{l}\text { Fuel region } \\
\end{array}$} & \multicolumn{2}{|c|}{ Monolith } \\
\hline Reactor thermal power & $5 \mathrm{MWth}(2 \mathrm{MWe})$ & Monolith material & SS316 \\
\hline $\mathrm{UO}_{2}$ density & $10.52 \mathrm{~g} / \mathrm{cm}^{3}$ & Monolith density & $8.03 \mathrm{~g} / \mathrm{cm}^{3}$ \\
\hline U-235 enrichment & $19.75 \mathrm{wt} \%$ & Fuel holes per block & 352 \\
\hline Fuel channel hole OD & $1.425 \mathrm{~cm}$ & HP holes per block & 204 \\
\hline Fuel pellet OD & $1.412 \mathrm{~cm}$ & Top \& bottom axial reflector & $15 \mathrm{~cm}$ \\
\hline Fuel rod length & $150 \mathrm{~cm}$ & \multicolumn{2}{|l|}{ Reflector } \\
\hline Fuel-to-fuel pitch & $1.60 \mathrm{~cm}$ & Side reflector material & $\mathrm{Al}_{2} \mathrm{O}_{3}$ \\
\hline Fuel-to-HP pitch & $1.60 \mathrm{~cm}$ & Alumina density & $3.9 \mathrm{~g} / \mathrm{cm}^{3}$ \\
\hline Gas filling gap & Helium & Side reflector outer radius & $77.85 \mathrm{~cm}$ \\
\hline Number of fuel rods in-core & 2,112 & \multicolumn{2}{|c|}{ Control rods } \\
\hline \multicolumn{2}{|c|}{ Heat pipe } & Number of control drums & 12 \\
\hline Number of HPs in core & 1,224 & Drum outer diameter & $25 \mathrm{~cm}$ \\
\hline HP hole diameter & $1.575 \mathrm{~cm}$ & Drum axial length & $200 \mathrm{~cm}$ \\
\hline HP-to-HP pitch & $2.7713 \mathrm{~cm}$ & Control material & $\mathrm{B}_{4} \mathrm{C}$ \\
\hline HP working fluid & Potassium & $\mathrm{B}_{4} \mathrm{C}$ density & $2.51 \mathrm{~g} / \mathrm{cm}^{3}$ \\
\hline
\end{tabular}

\subsubsection{PROTEUS Modeling}

For systematic verification of PROTEUS solutions, multiple benchmark problems developed based on the MegaPower reactor specification include a fuel pin, a unit cell, 2D and 3D cores with different control material locations in the control drums. For comparison of PROTEUS solutions, the SERPENT Monte Carlo code was run for those benchmark problems as well. 
Multigroup cross sections for PROTEUS can be generated in a couple of ways. Cross sections can be provided in the ISOTXS format from external codes such as $\mathrm{MC}^{2}-3$ or Monte Carlo codes, or they can be calculated on-the-fly inside the code using the cross section API [22] based on the subgroup or resonance table method. In this study, we generated multigroup cross sections using SERPENT whose output were converted to the ISOTXS format using GenISOTXS. Since the micro reactor problems of concern are fast neutron dominant systems as shown in Figure 3-1, the 33-group multi-group structure [12] that is typically used for fast reactor analysis was employed for multigroup cross section generation. For this preliminary study, cross sections were generated from the 2D core calculation with SERPENT, even though an accuracy issue of cross sections is expected for application to fast reactor systems because of inaccurate anisotropic scattering moments generated from SERPENT. [10] In order to reduce computation resources for 3D calculations, a 17-group structure was devised based on the 33-group structure, removing some low energy grids and making lethargies double at some high energies. Further analysis would be required to determine an optimized group structure in terms of performance and accuracy for this specific problem.

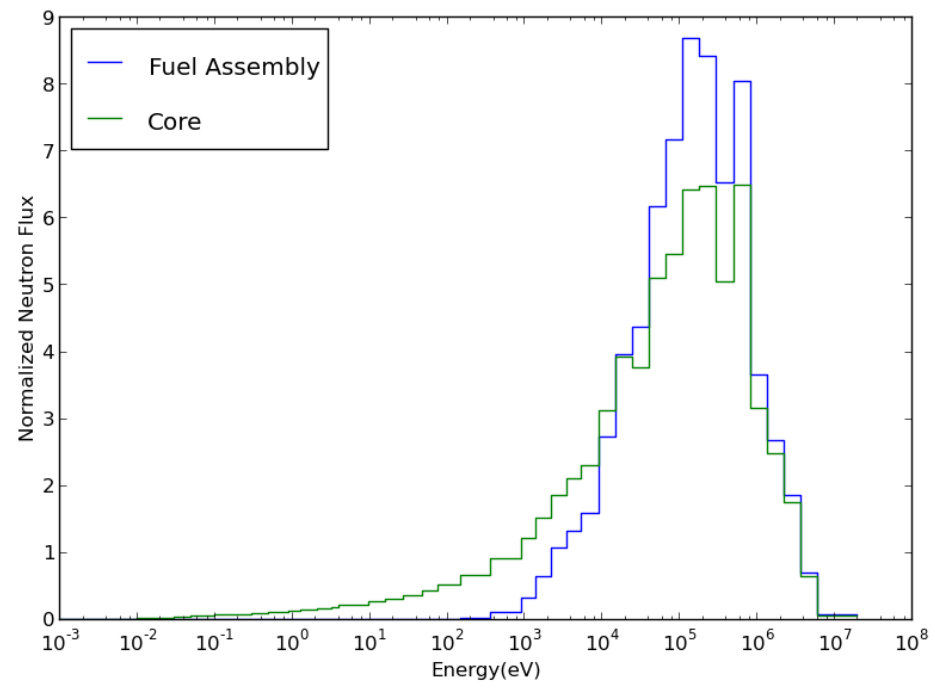

Figure 3-1. Neutron Flux Spectra of MegaPower Fuel Assembly and Core

The PROTEUS package includes the mesh generation toolkit that Argonne developed to allow users to quickly generate meshes for hexagonal or Cartesian geometry problems that are often applicable for many light water reactor and fast reactor problems. Since the micro reactor problems in this study are irregular-geometry cores, the mesh generation was conducted using the CUBIT geometry and mesh generation code and the PROTEUS mesh toolkit. Even though the meshes for the whole core can be generated using CUBIT only, but meshes of the core components were separately generated with CUBIT which were then merged to construct the whole core mesh using the PROTEUS mesh toolkit in order to make it easier to generate meshes for such a complex-geometry problem: i.e., six fuel blocks, a hex geometry at the center, and an out-core were generated separately using CUBIT and merged using the mesh toolkit. For simplicity, gaps between assemblies were ignored in the benchmark models. Note that when generating meshes with CUBIT the size of circular geometries must be adjusted to make their meshed areas match with the corresponding original geometry areas. 
Figure 3-2 shows the meshes generated for a 2D whole core as well as a fuel pin and a unit cell. Since we used the MOC solver of PROTEUS for this study, a 2D mesh is sufficient for the MOC solver, based on a 2D extruded mesh, for which a 3D problem is conducted by assigning compositions to axial planes via the composition assignment input of the code. For brevity, the PROTEUS-MOC solver is simply referred to PROTEUS hereafter.

In order to see a control material effect with different locations of control material in the 12 control drums, four cases were modelled as shown in Figure 3-3: in Cases A to D, the control material is placed at the location where it is the farthest from, half-ways with different patterns, and the closest to the core, respectively. Only cases with symmetric locations of control material in the drums are analyzed in this study.

In PROTEUS calculations, a Legendre-Tchebychev cubature of order $\mathrm{L}_{5} \mathrm{~T}_{15}$ (192 directions / $4 \pi$ ) was used for angular, with which based on preliminary studies solutions are almost converged with respect to angle. An anisotropic scattering order of $\mathrm{P}_{2}$ was selected. The $2 \mathrm{D}$ whole-core problem DOFs include 224,092 elements, 33 groups, and 192 directions/4 $\pi$ to deal with in terms of space, energy, and angle, respectively. For the 3D problem, total 19 or 34 axial planes should be added to the 2D problem DOFs: i.e., axially 2 planes for each of top and bottom reflectors (15 $\mathrm{cm}$ high each) and 15 or 30 planes for the fuel region $(150 \mathrm{~cm}$ high).
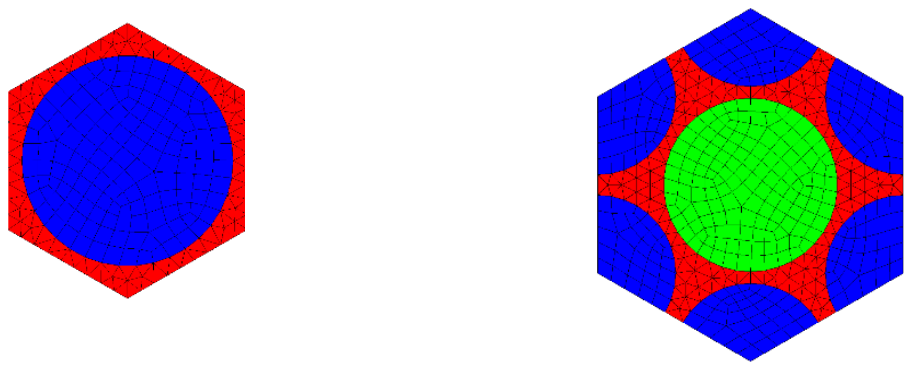

Fuel Pin

Unit Cell
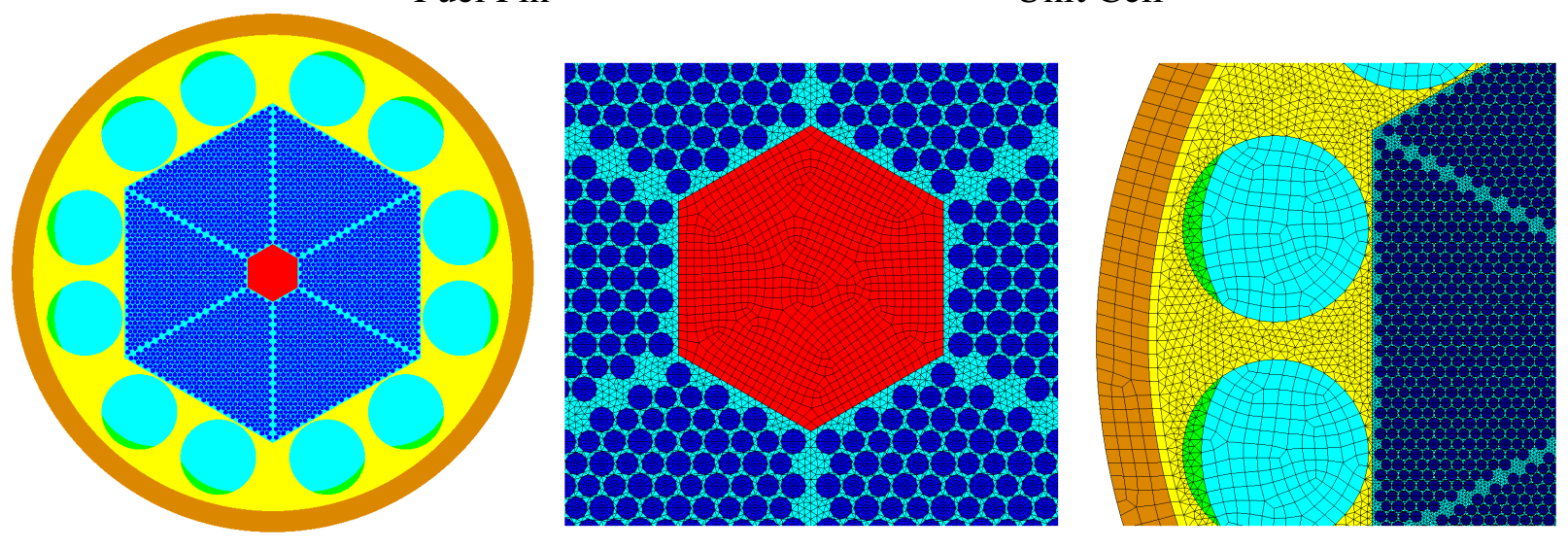

Figure 3-2. Geometry and Mesh Generation for the MegaPower Reactor 


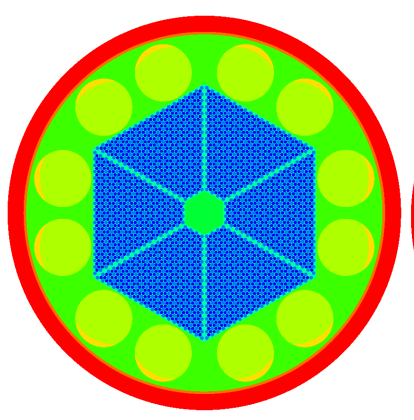

Case A

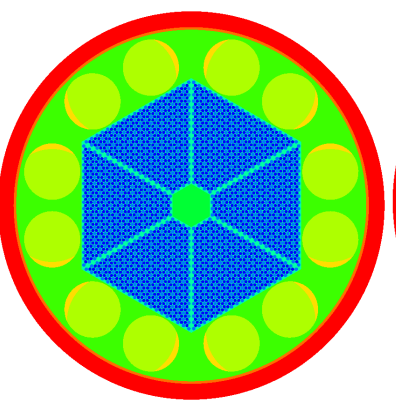

Case B

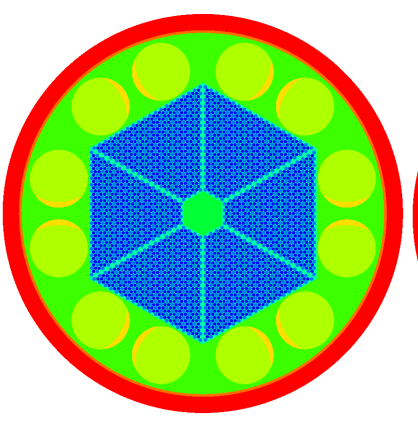

Case C

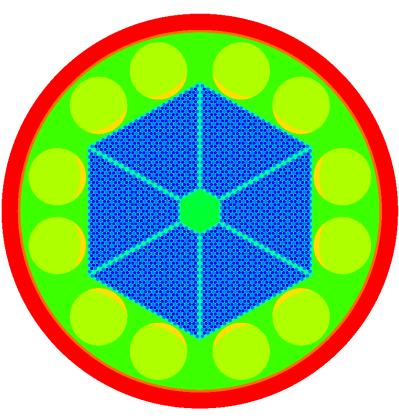

Case D

Figure 3-3. Benchmark Problems with Different Control Material Locations in the Control Drums

Note that PROTEUS includes the coarse mesh finite difference (CMFD) capability for accelerating solution convergence, but it could not be used in this calculation because the problem geometry is irregular. The CMFD acceleration for an irregular geometry problem is under development.

\subsubsection{PROTEUS Solutions}

In order to check whether eigenvalue solutions of the core configurations under tests are reasonable, core calculations were performed using SERPENT with ENDF/B-VII.1 and VII.0 data and the temperatures of all $300 \mathrm{~K}$ or hot full power (HFP: $1,200 \mathrm{~K}$ for the fuel rod regions and $900 \mathrm{~K}$ for the rest). Table 3-2 shows that eigenvalues with ENDF/B-VII.1 are $90-233$ pcm higher than those with ENDF/B-VII.0 and the 3D core eigenvalues with HFP are reasonable, indicating a core eigenvalue of 0.95271 for Case $\mathrm{D}$ where the control materials are located to the closest to the core. Note that the cases with all $300 \mathrm{~K}$ were used for the solution comparison of PROTEUS and SERPENT,

For PROTEUS simulations, cross sections were generated from SERPENT or MC ${ }^{2}-3$. When using SERPENT for the cross section generation, SERPENT outputs were processed with GenISOTXS to produce the cross sections in the ISOTXS format. To accurately account for the self-shielding effect of cross sections at each region, the cross sections of a pin, a unit cell, a FA, and $2 \mathrm{D}$ and $3 \mathrm{D}$ cores were generated from each representative case: i.e., the 2D Case A was used to generate cross sections for all $2 \mathrm{D}$ and $3 \mathrm{D}$ cases. As $\mathrm{MC}^{2}-3$ is not able to model the $2 \mathrm{D}$ core explicitly, the 1D cylindrical model representing the 2D core was used for the cross section generation.

Table 3-3 shows eigenvalue solutions from SERPENT and PROTEUS. As expected, core eigenvalues decrease as the control material turns toward the core in the order of Cases A, B, C, and D. The control material worth of Case $\mathrm{C}$ was larger than Case B. For most cases, differences in eigenvalue between the two codes are within $250 \mathrm{pcm}$, except for Case D in which the control material is located at the closest to the core. In Case D, a relatively larger gradient of neutron fluxes are generated radially and azimuthally due to the $\mathrm{B}_{4} \mathrm{C}$ absorbers right beside the fuel blocks. The trend is shown that differences in eigenvalue between the two codes are getting larger as the control material is turning toward the core. Unlike the solutions with the SERPENT cross sections, the PROTEUS eigenvalue solutions with the $\mathrm{MC}^{2}-3$ cross sections had good agreement even for Case D. Overall, the core eigenvalues agreed well with the SERPENT solutions within 
$144 \mathrm{pcm}$. In addition, no trend was shown that eigenvalue differences are getting larger with changing the locations of control material toward the core.

The large eigenvalue differences shown in Case D could be attributed to the inaccurate cross sections generated from SERPENT for fast spectrum systems. In SERPENT, all high-order scattering moments are generated not with corresponding angular moment weighting but with scalar flux weighting. Therefore, those inaccurate high-order scattering moments may affect the solution accuracy noticeably for fast reactor problems where the contribution of the neutron anisotropy and leakage effect to solutions is significant. This is currently a typical issue of the cross sections generated from most Monte Carlo codes.

Figure 3-4 shows the comparison of the axial relative power profiles produced from SERPENT and PROTEUS with the SERPENT or $\mathrm{MC}^{2}-3$ cross sections, indicating good agreement between them. The PROTEUS solutions with the $\mathrm{MC}^{2}-3$ cross sections produced slightly larger errors than those with the SERPENT cross sections at the core top and bottom boundaries.

The neutron flux solutions produced from PROTEUS depending upon the change of control material locations show a typical trend of changes with different energy groups and regions, as can be seen in Figure 3-5 where neutron fluxes from Cases A and D are illustrated at two different energies. One can clearly see the reduction of neutron fluxes near the control material turned toward the core. Figure 3-6 shows the flux distributions of the 3D benchmark problem of Case A. For the 3D core models, top and bottom reflector geometries with a thickness of $15 \mathrm{~cm}$ each are the same, except that the control material and drum exists in the top reflector only, as illustrated in the composition figure (left) in Figure 3-6.

Table 3-2. Eigenvalues of MegaPower Test Cases from SERPENT

\begin{tabular}{|c|c|c|c|}
\hline \multirow{2}{*}{ Case } & \multicolumn{3}{|c|}{ SERPENT } \\
\cline { 2 - 4 } & ENDF/B-VII.1,300K & ENDF/B-VII.0,300K & $\begin{array}{c}\text { ENDF/B-VII.1, } \\
\text { HFP* }\end{array}$ \\
\hline Fuel Pin & $1.41059 \pm 0.00009$ & $1.40940 \pm 0.00010$ & \\
Unit Cell & $1.37507 \pm 0.00009$ & $1.37125 \pm 0.00009$ & \\
Fuel Assembly & $1.34817 \pm 0.00009$ & $1.34452 \pm 0.00011$ & \\
\hline 2D Core A & $1.12696 \pm 0.00024$ & $1.12463 \pm 0.00024$ & $1.11199 \pm 0.00023$ \\
B & $1.10652 \pm 0.00026$ & $1.10517 \pm 0.00025$ & $1.08235 \pm 0.00027$ \\
C & $1.09202 \pm 0.00026$ & $1.09042 \pm 0.00026$ & $1.06259 \pm 0.00026$ \\
D & $1.04829 \pm 0.00029$ & $1.04739 \pm 0.00028$ & $1.00155 \pm 0.00029$ \\
\hline B & $1.06793 \pm 0.00017$ & $1.06584 \pm 0.00017$ & $1.05689 \pm 0.00016$ \\
C & $1.04942 \pm 0.00017$ & $1.04695 \pm 0.00017$ & $1.02786 \pm 0.00017$ \\
D & $1.03675 \pm 0.00019$ & $1.03349 \pm 0.00018$ & $1.00862 \pm 0.00019$ \\
\hline
\end{tabular}

* HFP: $1200 \mathrm{~K}$ for fuel and $900 \mathrm{~K}$ for the rest 
Table 3-3. Eigenvalue and Control Worth Comparison between SERPENT and PROTEUS for MegaPower Benchmark Cases

\begin{tabular}{|c|c|c|c|c|}
\hline \multirow{2}{*}{ Case } & \multicolumn{4}{|c|}{ PROTEUS } \\
\hline & ENDF/B-VII.1 (SERPENT XS) $)^{1)}$ & ENDF/B-VII.0 (MC (M XS $\left.^{2}\right)$ \\
\cline { 2 - 5 } & $\Delta \mathrm{k}, \mathrm{pcm}$ & CR Worth (Diff.) & $\Delta \mathrm{k}, \mathrm{pcm}$ & CR Worth (Diff.) \\
\hline Fuel Pin & 4 & & 187 & \\
Unit Cell & 169 & & 51 & \\
Fuel Assembly & -93 & & & \\
\hline 2D Core A & -50 & & 61 & \\
B & 73 & $1,639(-6.0 \%)$ & -96 & $1,566(8.1 \%)$ \\
C & 134 & $2,839(-5.4 \%)$ & -99 & $2,790(4.7 \%)$ \\
D & 530 & $6,659(-7.8 \%)$ & -38 & $6,557(1.3 \%)$ \\
\hline BD Core A & 134 & & -41 & \\
C & 256 & $1,652(-3.6 \%)$ & -127 & $1,613(4.9 \%)$ \\
D & 250 & $2,816(-2.1 \%)$ & -144 & $2,838(3.5 \%)$ \\
& 616 & $6,706(-6.6 \%)$ & -20 & $6,737(-0.2 \%)$ \\
\hline
\end{tabular}

1) Compared to the SERPENT results with EDNF/B-VII.1

2) Compared to the SERPENT results with EDNF/B-VII.0

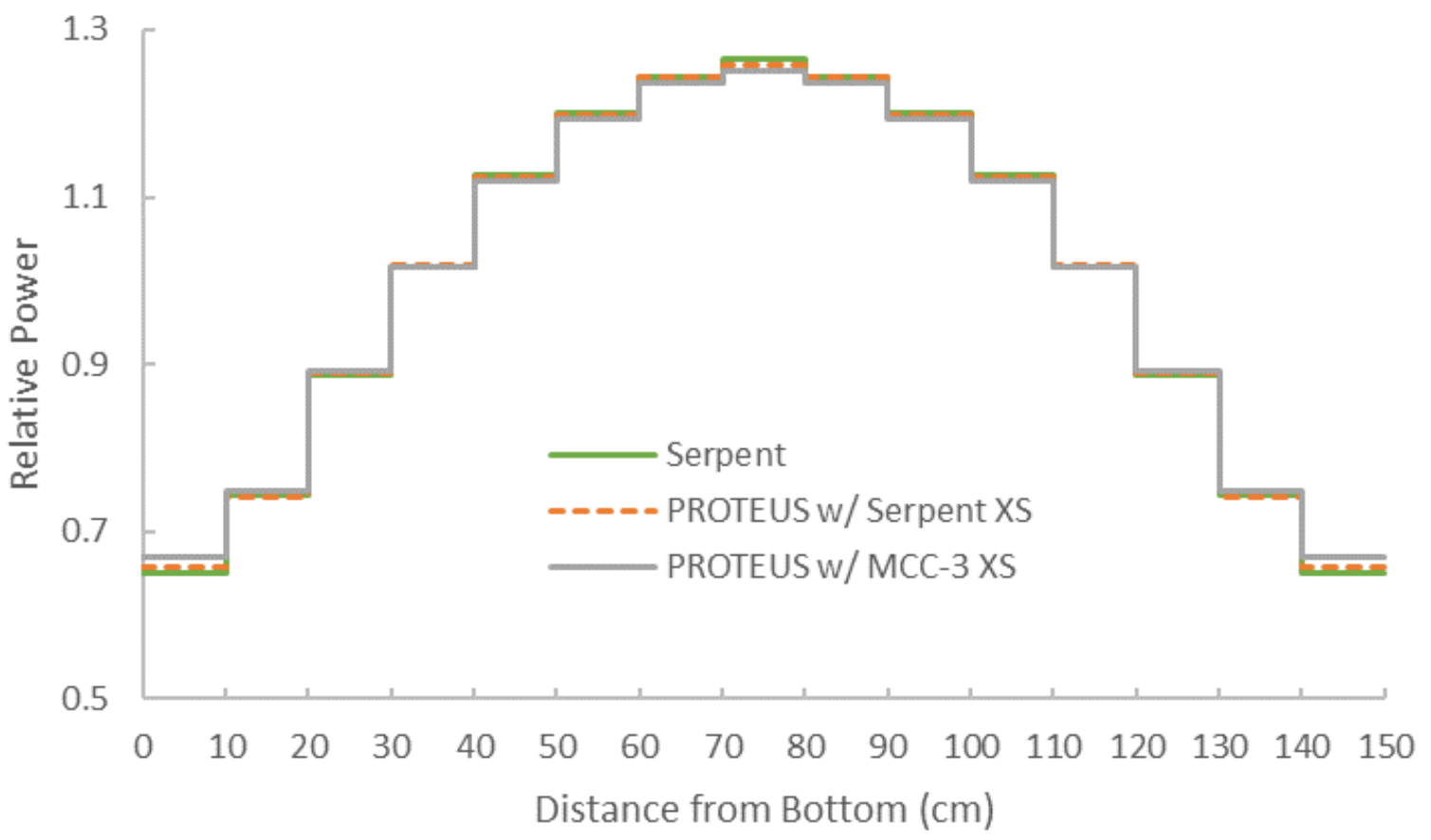

Figure 3-4. Comparison of Axial Power Profiles between SERPENT and PROTEUS for 3D Core Case A 

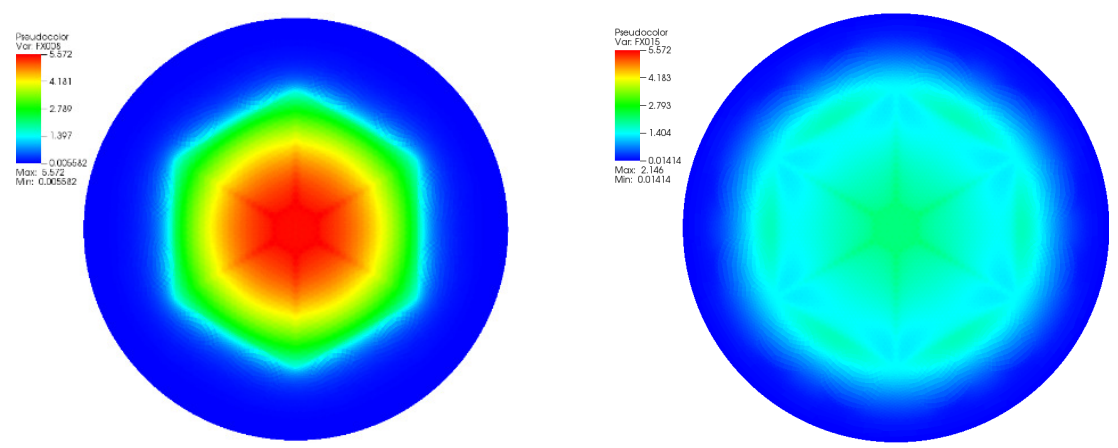

Case A
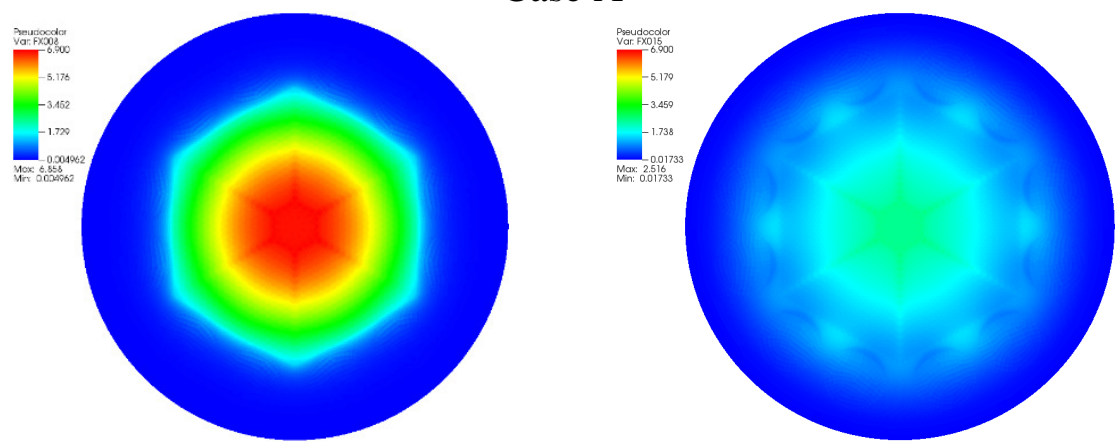

Case D
$0.3-0.5 \mathrm{MeV}$
$9-15 \mathrm{keV}$

Figure 3-5. Neutron Fluxes of MegaPower with Different CR Locations (Cases A and D)

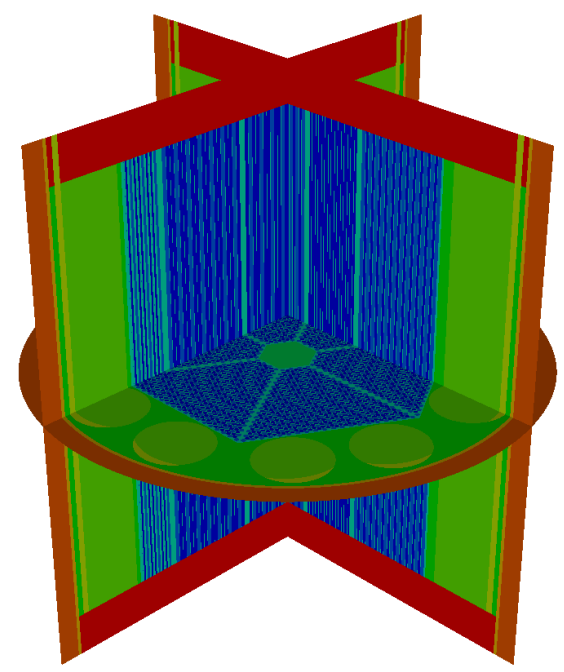

Composition

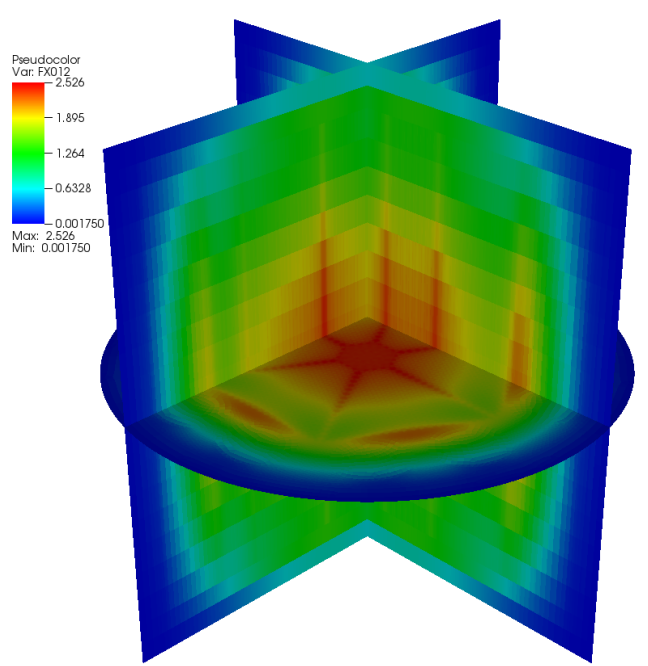

Neutron Flux at $9-15 \mathrm{keV}$

Figure 3-6. Neutron Fluxes of 3D Core Case A 


\subsection{High-Temperature Gas-cooled Micro Reactor}

\subsubsection{Core Description}

HolosGen proposes a highly innovative micro-reactor concept targeting both civilian and military applications. It consists of an advanced helium-cooled gas reactor using a turbojet-type turbine and compressor to achieve a highly condensed reactor that fits into a commercial ISO container $\left(2.34 \times 2.34 \mathrm{~m}^{2}\right.$ with $12.19 \mathrm{~m}$ long). [4] The Holos-Quad concept being considered for civilian applications will generate a thermal power of $22 \mathrm{MW}$, using four Subcritical Power Modules (SPMs) that fit into a 40-foot ISO container, as shown in Figure 3-7. It is a hightemperature gas-cooled reactor concept using TRISO fuel distributed in graphite hexagonal blocks, cooled with helium gas at $7 \mathrm{MPa}$ and a high outlet temperature of $650^{\circ} \mathrm{C}$ or higher.
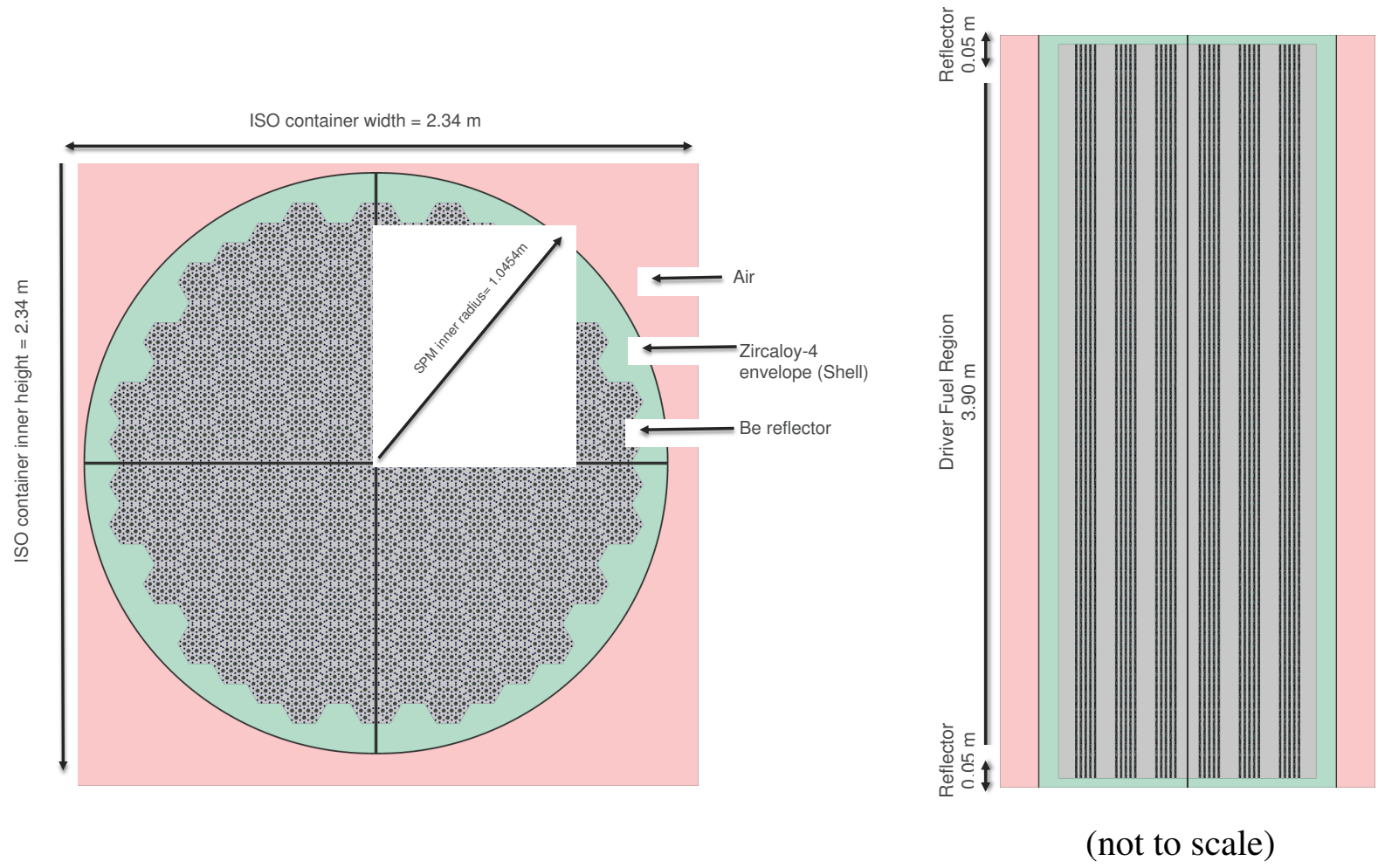

Figure 3-7. Holos-Quad Core Layout

Currently, only one reactivity control system is considered: the capability to manage reactivity by moving SPMs apart and increasing the neutron leakage. Additional emergency shutdown system, which will also be used for transportation, considers addition of a hafnium blade between the four SPMs. Each SPM is wrapped inside a shell of zircaloy-4. The purpose of the shell is mainly to avoid air interacting with graphite. Its purpose is not structural and it will not be subjected to excessive stress. The core weight will be held via structural components in the coolant sleeves and a solid structure outside the fuel cartridge.

For the full-core analysis, the following five sets of eigenvalue calculations were performed: [4] 
1. Reference k-eff for the four SPMs attached together. All cross-section temperatures are $800 \mathrm{~K}$, except for $900 \mathrm{~K}$ for fuel (UCO).

2. The reactivity worth of controlling SPM separation is assessed by moving 3 SPMs out of 4 , with maximum gap thickness of $21 \mathrm{~cm}$. The fuel temperature is $800 \mathrm{~K}$ (hot shutdown).

3. The shutdown margins are assessed with k-eff of Holos-Quad with maximum gap thickness between each of the 4 SPMs and insertion of Hafnium blade $(1 \mathrm{~cm}$ thick, 13.203 $\mathrm{g} / \mathrm{cm}^{3}$ ). The fuel and structure temperatures are $300 \mathrm{~K}$ (cold shutdown).

4. The moderator density coefficient (MDC) is calculated by reducing the graphite density of the fuel pin matrix and the graphite block by $1 \%$ (density of Be reflector is not changed).

5. The Doppler temperature coefficient (DTC) is calculated by increasing the temperature of the UCO fuel by $300 \mathrm{~K}$ (to $1,200 \mathrm{~K}$ ).

\subsubsection{PROTEUS Geometry and Mesh}

Geometry and mesh of Holos benchmark cores for PROTEUS were generated using CUBIT and the in-house mesh toolkit. 2D meshes were generated to solve 2D and 3D core problems with PROTEUS-MOC, which solves problems based on the extruded geometry. Since the Holos core is based on a non-standard geometry requiring a significant effort to deal with a whole-core geometry and mesh using CUBIT, we generated meshes for core components such as fuel assemblies and out-core regions using CUBIT and merged them using the in-house mesh toolkit to construct a whole core. This allowed us to save time and effort in generating geometries and meshes.
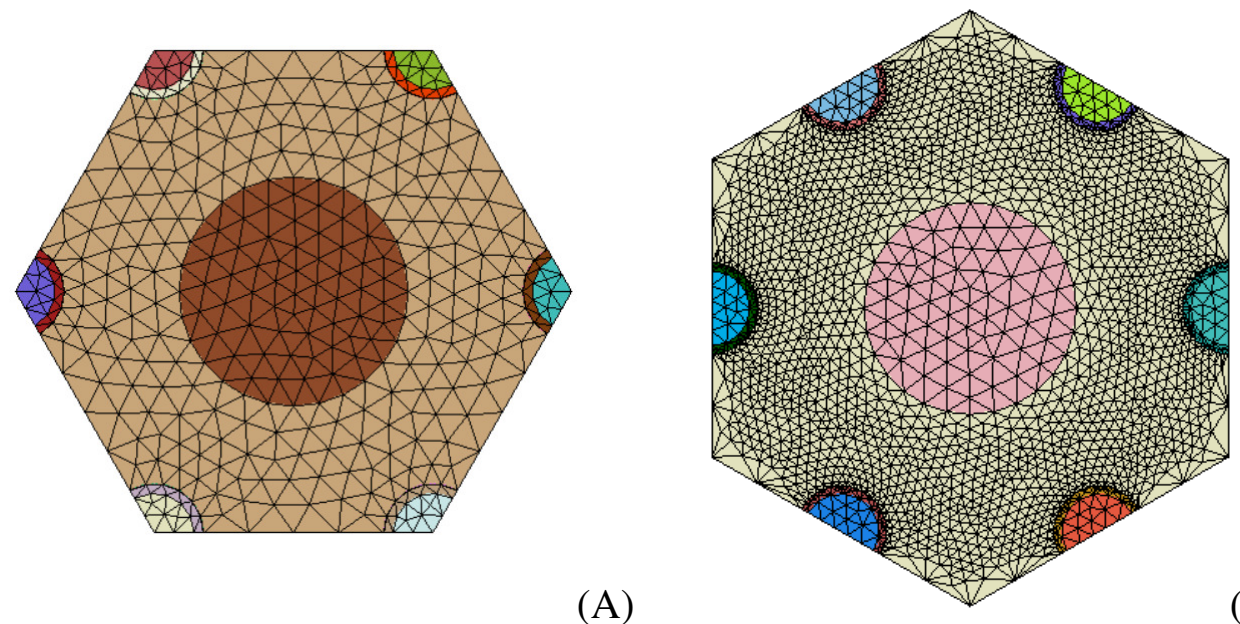

(A)

Figure 3-8. Unit Cell Benchmark Problems

Figure 3-8 shows the unit cell benchmark problems, among which configuration $\mathrm{B}$ (pitch = $3.4 \mathrm{~cm}$ ) was selected for comparison with Monte Carlo (SERPENT and OpenMC [23]) solutions even though configuration A (pitch $=1.7^{*} \mathrm{~cm}$ ) was more closely representing a unit cell of the fuel region of the core. Meshes were generated using CUBIT. 
In order to contruct a whole core geometry and mesh, at first set of assembly meshes were generated using CUBIT. Since the core is composed of partial fuel assemblies along the SPM boundaries as well as whole fuel assemblies, we generated geometry and mesh for partial fuel assemblies shown in Figure 3-9 as well. Those four types were used to construct the core composed of fuel assemblies only, by merging fuel assemblies using the in-house mesh toolkit. The resulting geometry is shown in Figure 3-9 (bottom left).

Geometry and mesh were generated for out-cores with different SPM conditions in the ISO container (no separation, full separation, and full separation with the control blade), as shown in Figure 3-10. A quarter core is constructed by merging the core and out-core components using the in-house mesh toolkit. Four quarter cores are merged to make a whole core. A whole core with asymmetric SPM separations, 3 SPM separation for example, is generated by merging 3 quarter cores with SPM separation and 1 quarter core with no SPM separation.
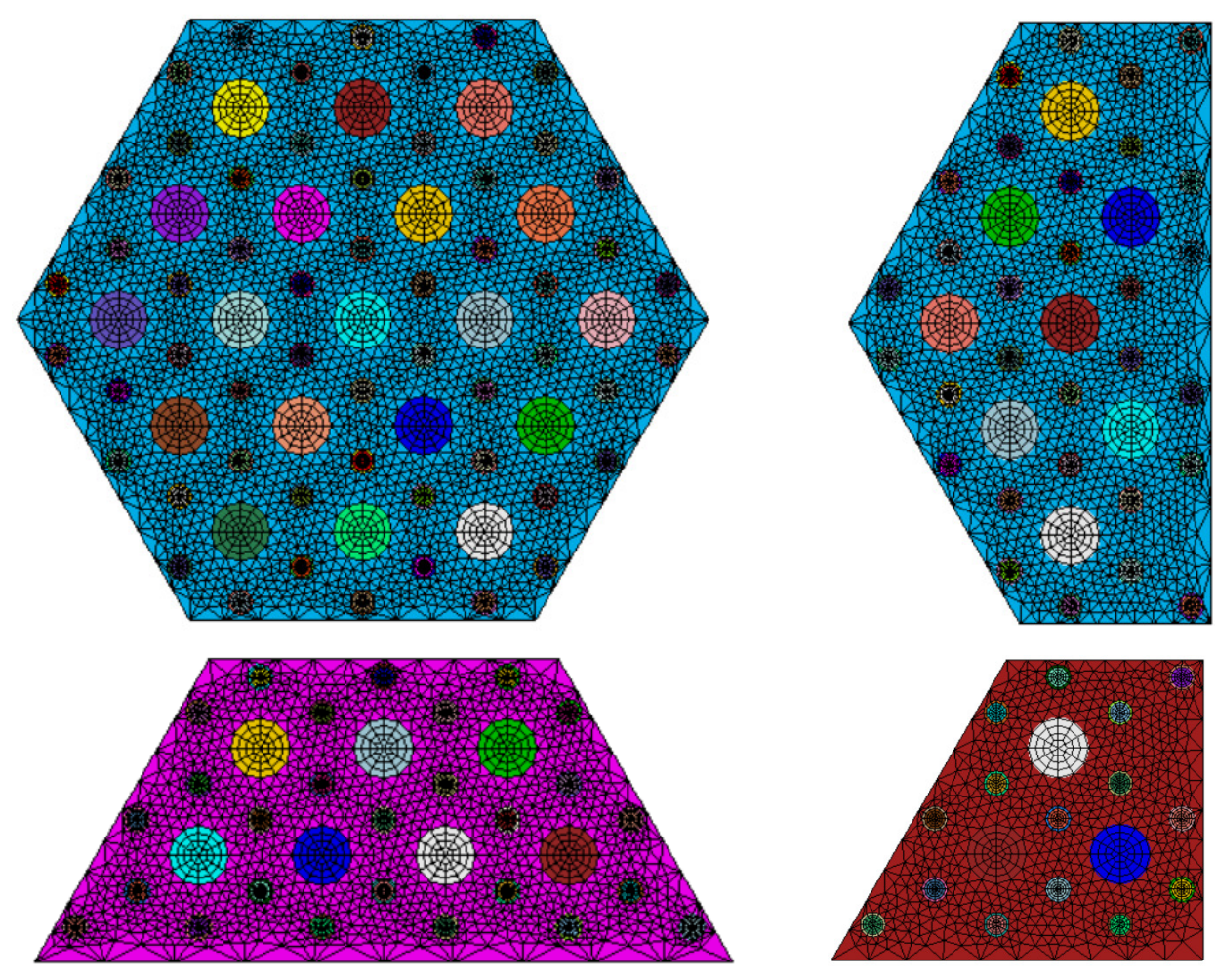

Figure 3-9. Fuel Assembly Meshes Generated from CUBIT

Part of the detailed meshes of a whole core are illustrated in Figure 3-11. As seen in the figure, meshes should be conformal between different material regions since PROTEUS does not handle non-conformal meshes. Both quad and triangle meshes were used. In particular, the meshed areas for fuel and coolant regions were slightly adjusted to preserve those of the actual regions. 

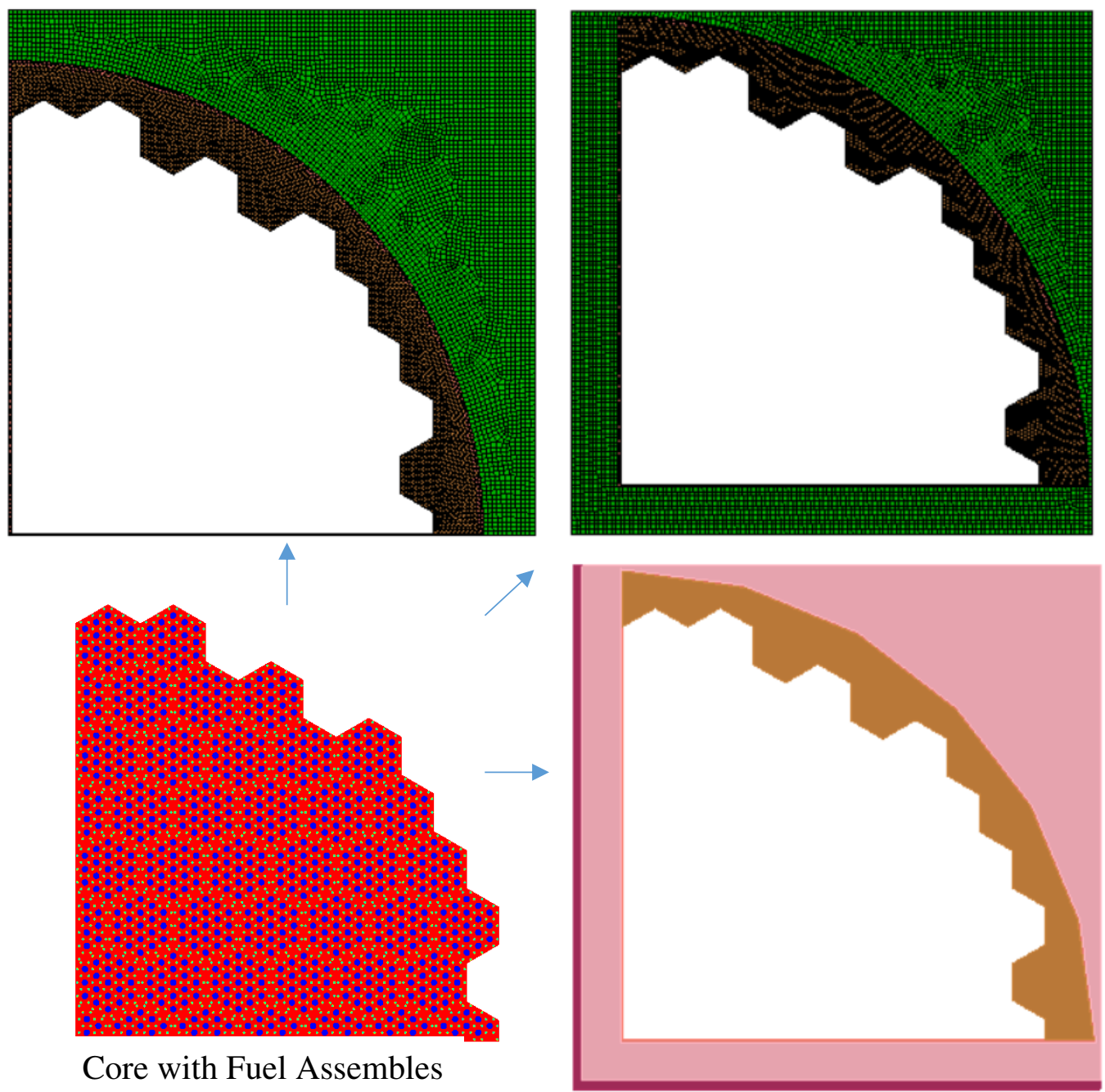

Figure 3-10. Core and Out-core Meshes Generated from CUBIT
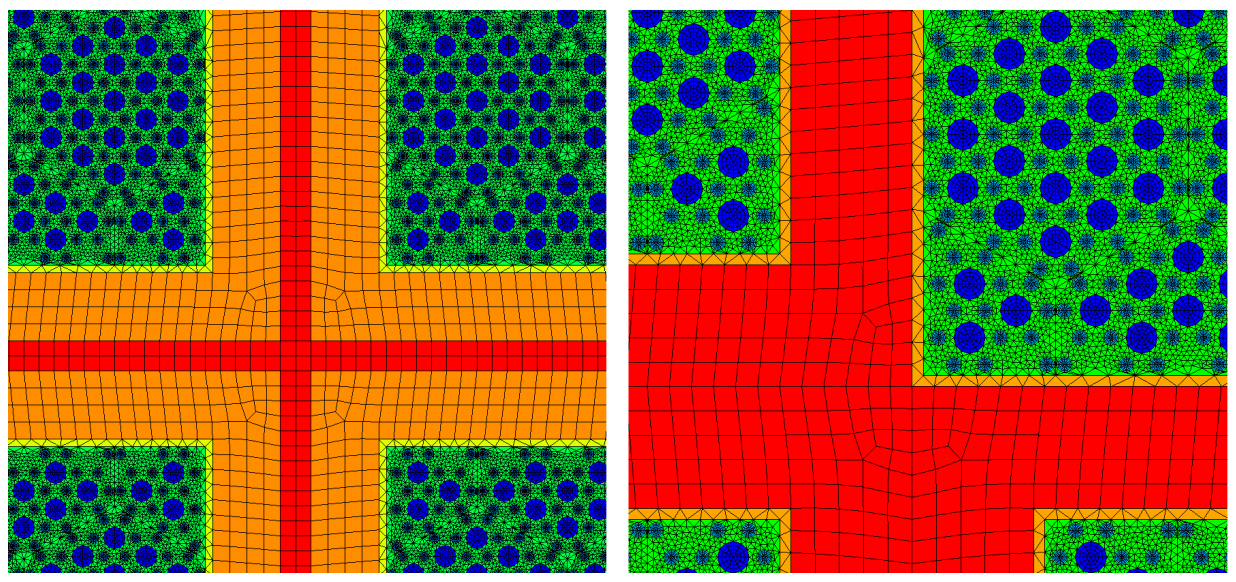

Figure 3-11. Details of Core Meshes 

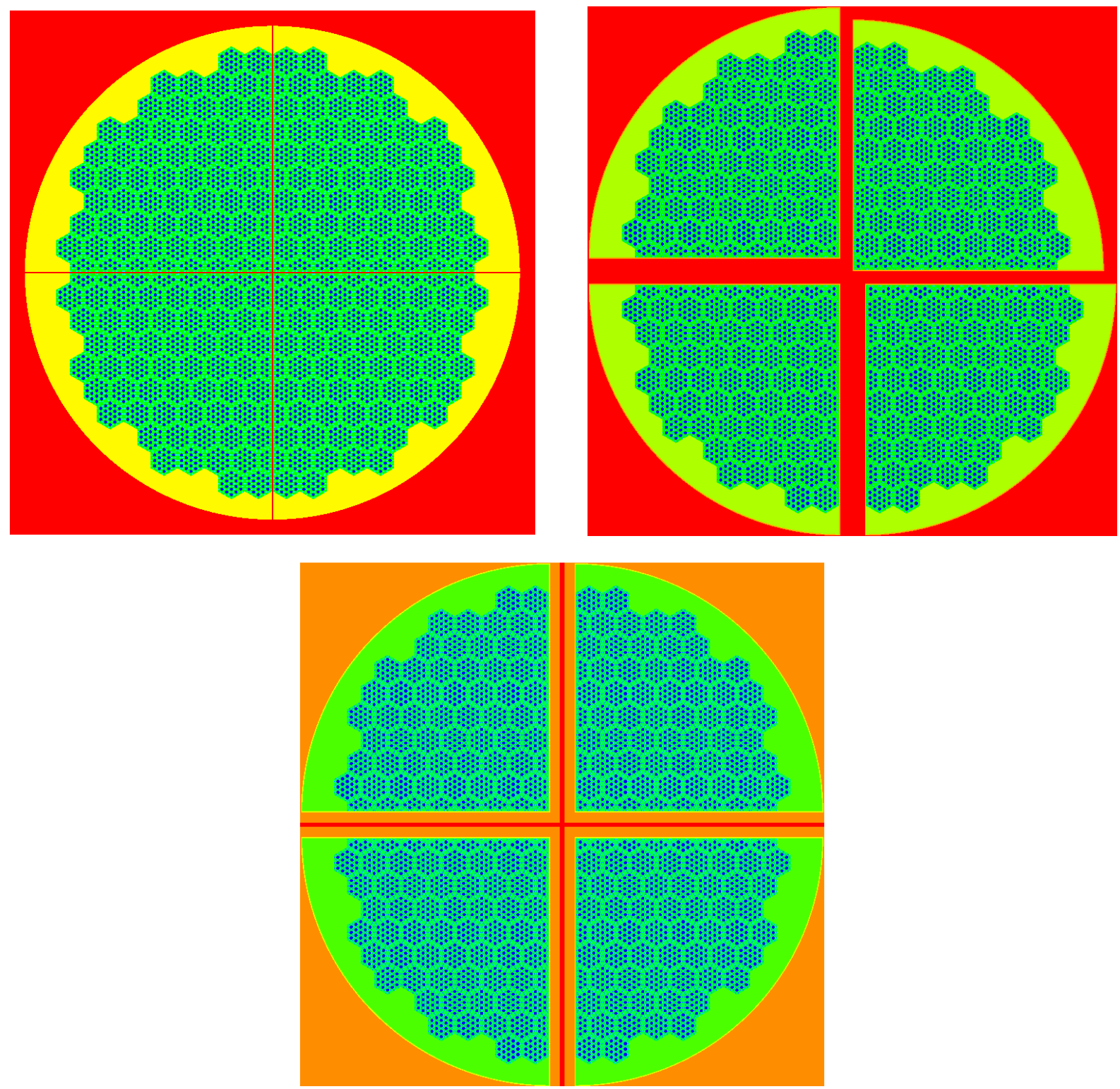

Figure 3-12. PROTEUS Core Models with Different SPM Configurations

Boundary surfaces (curves) for boundary conditions are specified using sidesets, and regions are defined as blocks with names such that materials and cross sections are assigned to regions for PROTEUS calculations. The PROTEUS core model obtained is shown in Figure 3-12.

\subsubsection{PROTEUS Cross Sections}

Cross sections were generated using SERPENT / GenISOTXS. The 2D core without gap between SPMs was calculated using SERPENT, and multi-group cross sections are extracted from SERPENT outputs using GenISOTXS. Multigroup cross sections would be different from regions even with the same composition because of different self-shielding effects, but we produced one representative set of cross sections for each region (i.e., fuel, coolant, graphite, reflector, control blade, and shell). 
The double heterogeneity effect due to TRISO particles was dealt with by SERPENT, not directly by PROTEUS. The 14-group cross sections based on the group structure listed in Table 3-4 were extracted. The neutron flux spectra for the Holos cores with different configurations are illustrated in Figure 3-13. The neutron flux spectra for cases 1, 2, 4, and 5 are similar to each other, which are typical neutron flux spectra for cores with graphite moderator. Case 3 in which 4 SPMs are fully separated and the $\mathrm{Hf}$ control blade is fully inserted at the center shows relatively a smaller amount of thermal cross sections due to the neutron absorption of the Hf control blade. In these benchmark calculations, we used 14 group cross sections generated from SERPENT for each calculation.

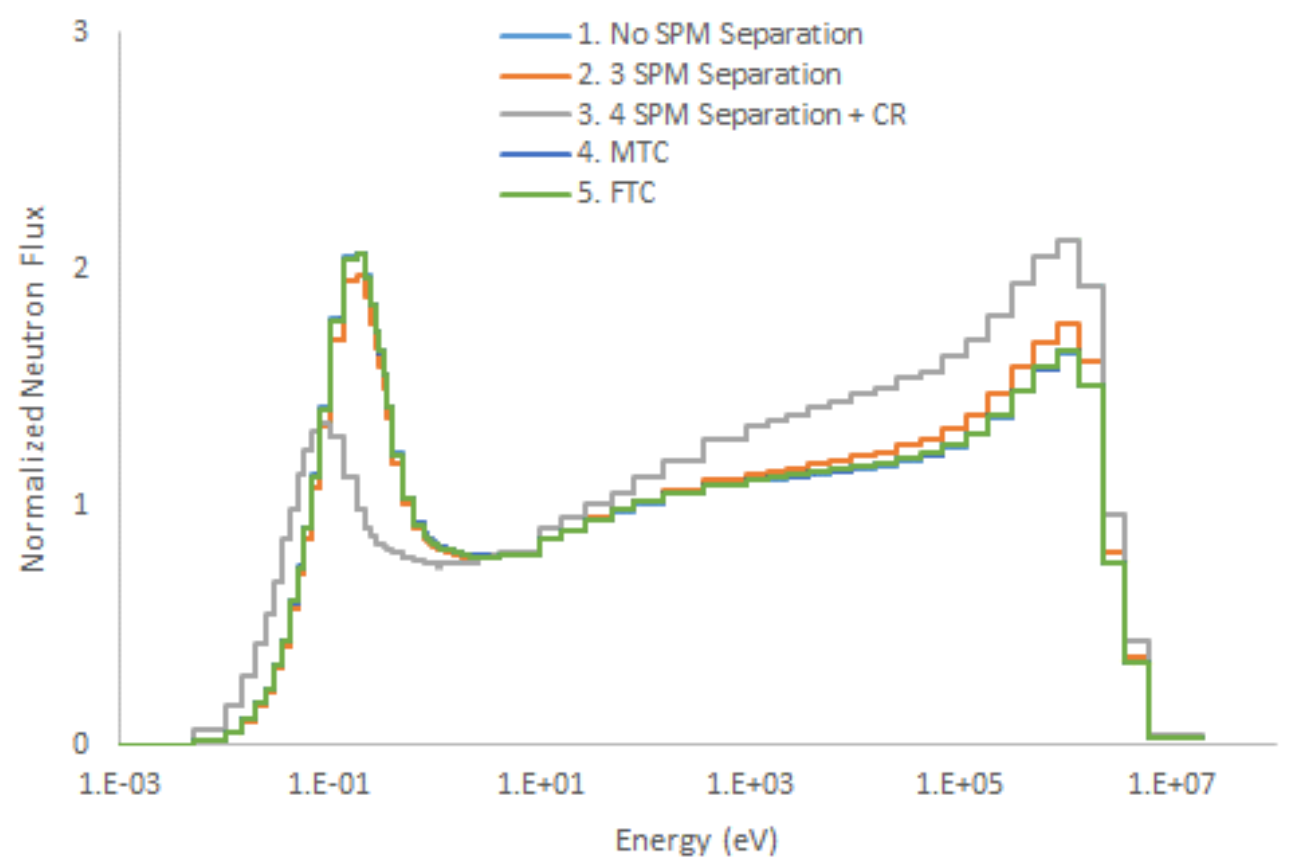

Figure 3-13. Normalized Neutron Flux Spectra of 3D Holos Core Configurations

Table 3-4. 14 Group Structure (Upper Energies)

\begin{tabular}{|c|c|c|c|c|c|c|c|}
\hline $\mathrm{G}$ & Energy $(\mathrm{eV})$ & $\mathrm{G}$ & Energy $(\mathrm{eV})$ & $\mathrm{G}$ & Energy $(\mathrm{eV})$ & $\mathrm{G}$ & Energy $(\mathrm{eV})$ \\
\hline 1 & $2.00000 \mathrm{e}+07$ & 2 & $1.35300 \mathrm{e}+06$ & 3 & $5.00000 \mathrm{e}+05$ & 4 & $9.11800 \mathrm{e}+03$ \\
\hline 5 & $3.67260 \mathrm{e}+02$ & 6 & $4.00000 \mathrm{e}-00$ & 7 & $1.50000 \mathrm{e}-00$ & 8 & $5.00000 \mathrm{e}-01$ \\
\hline 9 & $4.00000 \mathrm{e}-01$ & 10 & $3.00000 \mathrm{e}-01$ & 11 & $2.50000 \mathrm{e}-01$ & 12 & $1.80000 \mathrm{e}-01$ \\
\hline 13 & $1.00000 \mathrm{e}-01$ & 14 & $5.00000 \mathrm{e}-02$ & \multicolumn{5}{|r}{} \\
\cline { 1 - 5 } & &
\end{tabular}




\subsubsection{PROTEUS Solutions}

2D and 3D cores with different SPM conditions and different materials in the gap between SPMs were calculated with PROTEUS. For 2D cores, 332,449 elements, 80 angles $\left(\mathrm{L}_{3} \mathrm{~T}_{9}\right)$ or 128 angles $\left(\mathrm{L}_{3} \mathrm{~T}_{15}\right)$ per all directions, 14 groups were used, while for $3 \mathrm{D}$ cores with $400 \mathrm{~cm}$ high including $390 \mathrm{~cm}$ fuel region with $5 \mathrm{~cm}$ top and bottom axial reflectors, 21 axial planes per a half core were used.

Table 3-5 shows comparison of eigenvalues for the 2D calculations between SERPENT and PROTEUS. All eigenvalues are in good agreement between the two code solutions, except for case 3 in which the hafnium control blade is inserted. It was indicated that the MDC and FTC agree very well between SERPENT and PROTEUS. As a future work, further investigation would be necessary to figure out the sources of the difference observed in the calculations with Hf control blade.

Figure 3-14 and Figure 3-15 illustrate 2D thermal $(0.1-0.2 \mathrm{eV})$ and fast $(0.9 \mathrm{keV}-0.5 \mathrm{MeV})$ neutron flux distributions for case 2 (3 SPM separation) and case 3 (SPM separation with hafnium control blade), produced from PROTEUS. As seen in the figures, large thermal fluxes are shown in the reflector region of cases 2 and 3, and small thermal fluxes in the center regions of case 3 due to the presence of control blades.

Since all calculations except case 2 are quarter-core symmetric, quarter cores were modeled and calculated. Additionally, since the core is axially half-symmetric (the active core with $390 \mathrm{~cm}$ high and the top and bottom reflectors with $5 \mathrm{~cm}$ thick), half cores were modeled and calculated

for case 2. For verification, 3D cores were calculated using PROTEUS and compared with SERPENT solutions, as listed in Table 3-6.

For the selected calculations (cases 1 and 2), the 3D flux distributions produced from PROTEUS are shown in Figure 3-16 and Figure 3-17. The solutions indicate that the maximum group fluxes of cases 1 and 2 are $1.37 \times 10^{12}$ and $1.17 \times 10^{12} \# / \mathrm{cm}^{2} \cdot \mathrm{s}$, respectively, and the maximum relative powers are 2.19 and 1.46 . The maximum relative powers for the integrated $2 \mathrm{D}$ are approximately 1.72 and 1.38 , respectively. Therefore, the axial peak factors for cases 1 and 2 would be roughly 1.27 and 1.15 , respectively. 
Table 3-5. Benchmark Results for 2D Holos Core Problems

\begin{tabular}{|c|l|c|c|}
\hline Case & \multicolumn{1}{|c|}{ Description } & $\begin{array}{c}\text { SERPENT } \\
\mathrm{k}-\mathrm{eff} \pm \sigma \mathrm{pcm}\end{array}$ & $\begin{array}{c}\text { PROTEUS } \\
\Delta \mathrm{k}^{*}, \mathrm{pcm}\end{array}$ \\
\hline $\mathbf{1}$ & No SPM separation & $1.08191 \pm 14$ & -17 \\
\hline $\mathbf{2}$ & $\begin{array}{l}\text { Reactivity worth at hot shutdown of moving 3 SPMs out of 4, } \\
\text { with maximum gap thickness of 21 cm }\end{array}$ & $0.96731 \pm 17$ & -101 \\
\hline $\mathbf{3}$ & $\begin{array}{l}\text { Shutdown margins at cold shutdown with maximum gap } \\
\text { thickness of 21 cm between each of the 4 SPMs, and insertion } \\
\text { of Hafnium blade }\end{array}$ & $0.82282 \pm 17$ & -51 \\
\hline $\mathbf{4}$ & $\begin{array}{l}\text { Moderator density coefficient with reduced graphite density of } \\
\text { the fuel pin matrix and the graphite block by 1\% }\end{array}$ & $1.08008 \pm 14$ & -29 \\
\hline $\mathbf{5}$ & $\begin{array}{l}\text { Doppler coefficient calculated by increasing the temperature } \\
\text { of the UCO fuel by 300K }\end{array}$ & $1.07040 \pm 15$ & -84 \\
\hline $\mathbf{\rho}$ & $\begin{array}{l}\text { MDC (using cases 1 and 4, pcm/\%) } \\
\text { FTC (using cases 1 and 5, pcm/K) }\end{array}$ & -157 & -167 \\
\hline
\end{tabular}

$* \Delta \mathrm{k}$ : eigenvalue difference from SERPENT, $\mathrm{pcm}$

Table 3-6. Benchmark Results for 3D Holos Core Problems

\begin{tabular}{|c|l|c|c|}
\hline Case & \multicolumn{1}{|c|}{ Description } & $\begin{array}{c}\text { SERPENT } \\
\text { k-eff } \pm \sigma \mathrm{pcm}\end{array}$ & $\begin{array}{c}\text { PROTEUS } \\
\Delta \mathrm{k}^{*}, \mathrm{pcm}\end{array}$ \\
\hline $\mathbf{1}$ & No SPM separation & $1.06165 \pm 16$ & -218 \\
\hline $\mathbf{2}$ & $\begin{array}{l}\text { Reactivity worth at hot shutdown of moving 3 SPMs out of 4, } \\
\text { with maximum gap thickness of 21 cm }\end{array}$ & $0.92772 \pm 18$ & +187 \\
\hline $\mathbf{3}$ & $\begin{array}{l}\text { Shutdown margins at cold shutdown with maximum gap } \\
\text { thickness of 21 cm between each of the 4 SPMs, and insertion } \\
\text { of Hafnium blade }\end{array}$ & $0.80582 \pm 23$ & -63 \\
\hline $\mathbf{4}$ & $\begin{array}{l}\text { Moderator density coefficient with reduced graphite density } \\
\text { of the fuel pin matrix and the graphite block by 1\% }\end{array}$ & $1.05974 \pm 16$ & -227 \\
\hline $\mathbf{5}$ & $\begin{array}{l}\text { Doppler coefficient calculated by increasing the temperature } \\
\text { of the UCO fuel by 300K }\end{array}$ & $1.05083 \pm 15$ & -85 \\
\hline $\boldsymbol{\rho}$ & $\begin{array}{l}\text { MDC (using cases 1 and 4, pcm/\%) } \\
\text { FTC (using cases 1 and 5, pcm/K) }\end{array}$ & -170 & -156 \\
\hline
\end{tabular}

$* \Delta \mathrm{k}$ : eigenvalue difference from SERPENT, $\mathrm{pcm}$ 


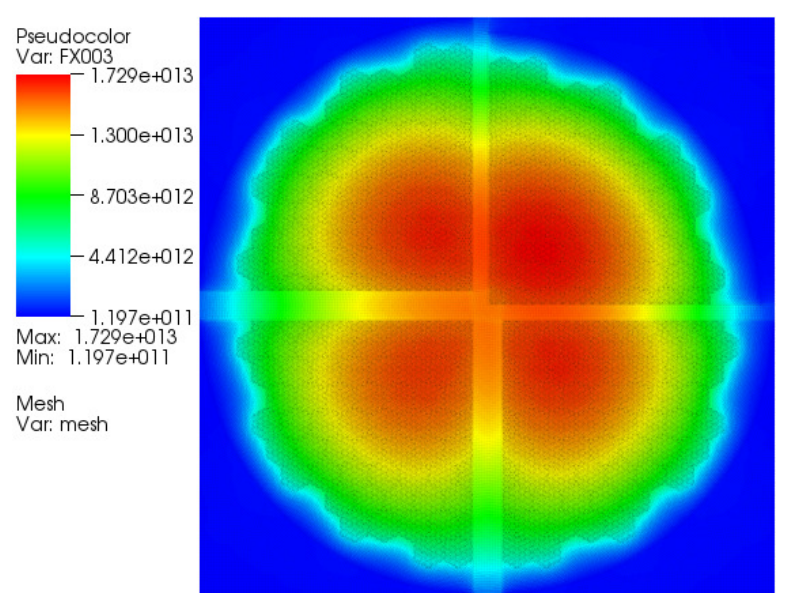

$0.9 \mathrm{keV}-0.5 \mathrm{MeV}$

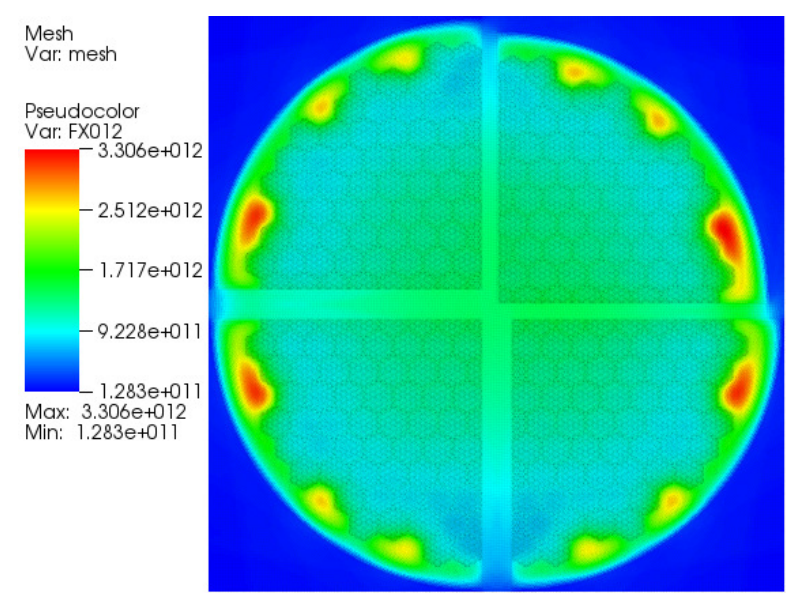

$0.1-0.2 \mathrm{eV}$

Figure 3-14. Thermal and Fast Flux Distributions of 2D Holos Cores for Case 2
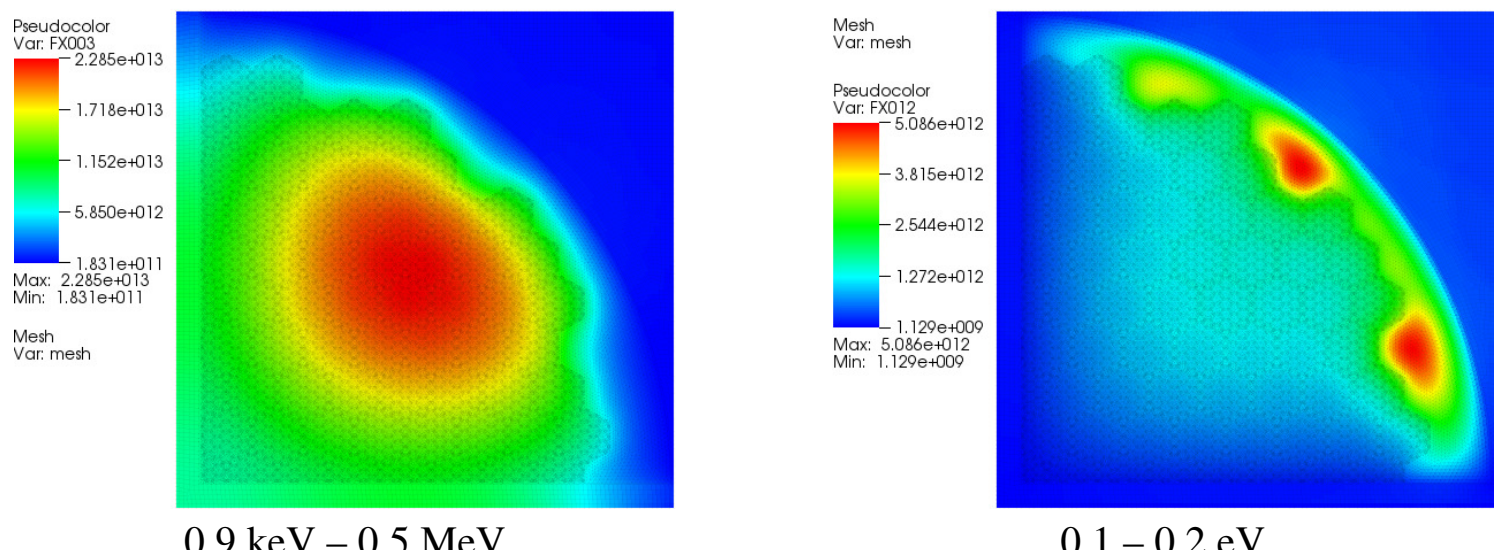

Figure 3-15. Thermal and Fast Flux Distributions of 2D Holos Cores for Case 3 

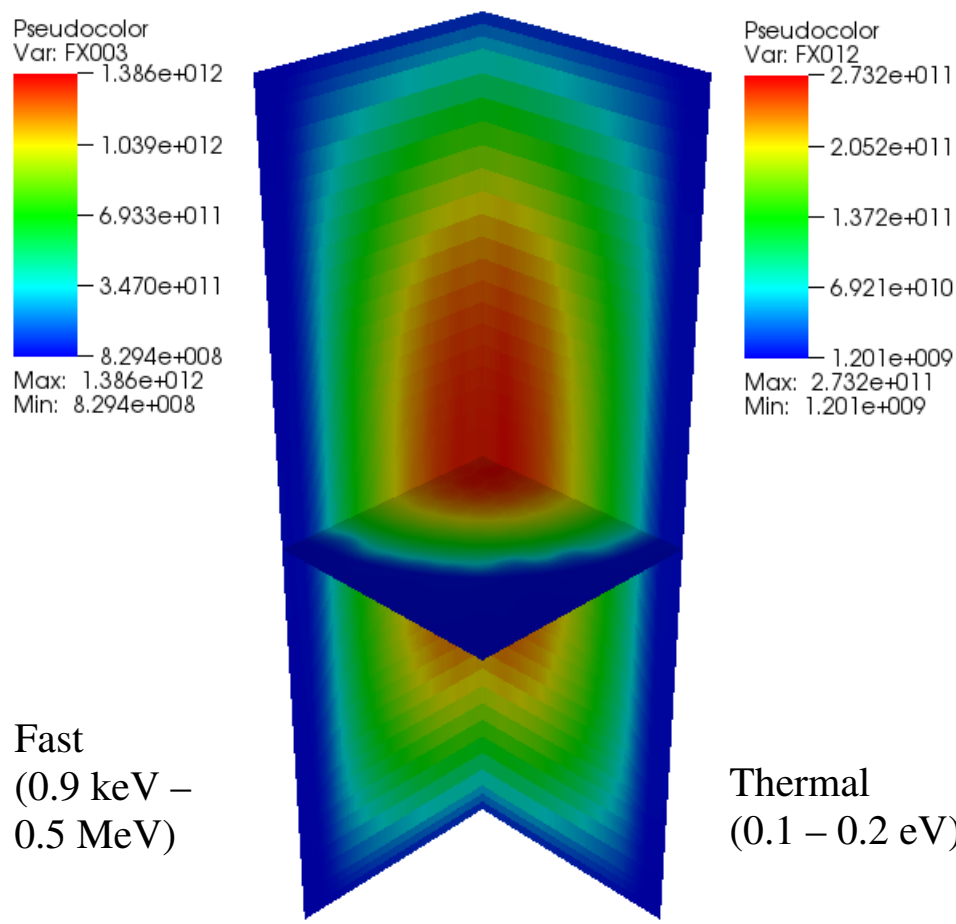

Min: $8.294 e+008$

Fast

$(0.9 \mathrm{keV}-$

$0.5 \mathrm{MeV})$

Thermal

$(0.1-0.2 \mathrm{eV})$

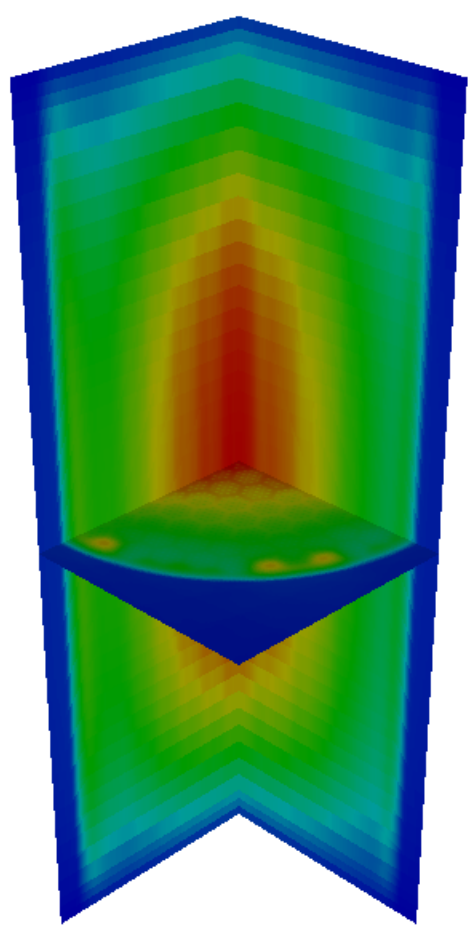

Figure 3-16. Neutron Flux Distributions of 3D Holos Core Calculation 1 (No Gap)
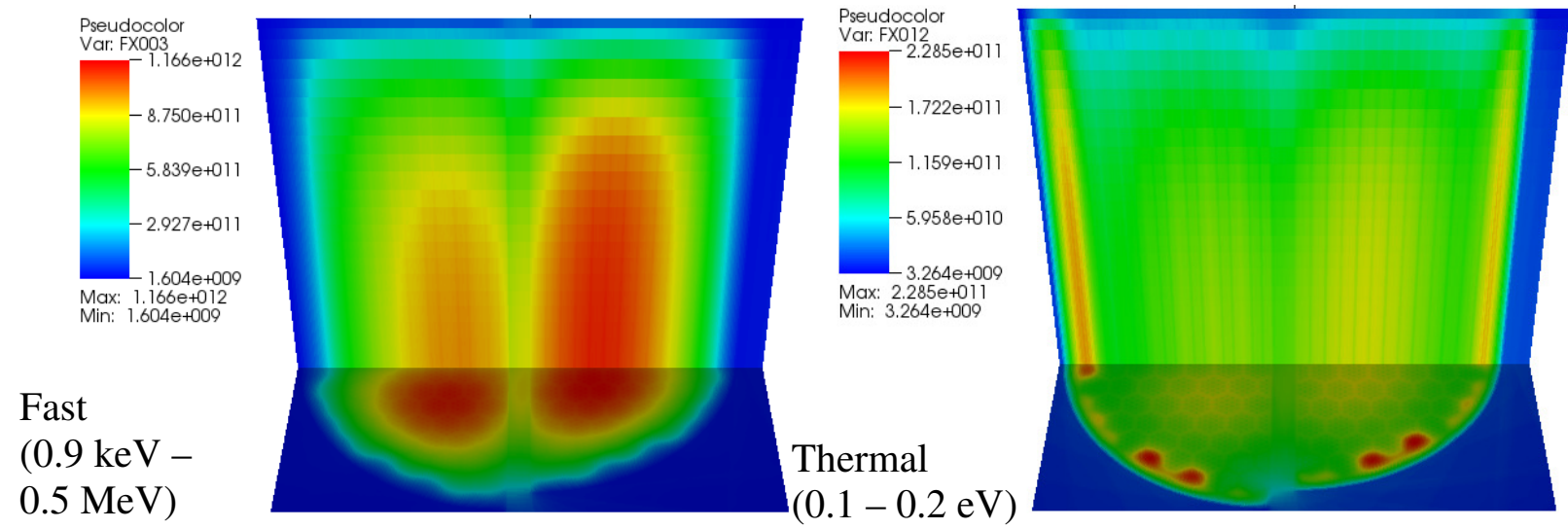

Figure 3-17. Neutron Flux Distributions of 3D Holos Core Calculation 2 (3 SPMs Separation) 


\section{Coupled Simulation of Micro Reactors}

\subsection{Coupled System of PROTEUS / FLUENT / ANLHTP}

For multiphysics simulation of micro reactors, three physics codes were coupled together as shown in Figure 4-1: PROTEUS for neutronics analysis, FLUENT for thermal analysis, and ANLHTP for heat pipe performance analysis. An efficient way of coupling different codes would be to develop a driver module and compile all codes together in order to control physics components by calling them directly and exchanging data required for individual physics calculations via data memories. Since, however, FLUENT is a commercial code which is difficult to compile with external codes, power and temperature data were transferred between PROTEUS and FLUENT via files. Additionally, Python-based external drivers were developed to coordinate the overall workflow including data transfer and to control individual calculation steps of the coupling of PROTEUS and FLUENT. Specifically for PROTEUS, the Python driver utilizes the Message Passing Interface (MPI) library to connect the PROTEUS solvers, which enables to control the neutronics calculation flow and transfer data through MPI communications. PROTEUS was updated to support the MPI-based Python driver, implementing the interface and data exchange routines for the Python driver.

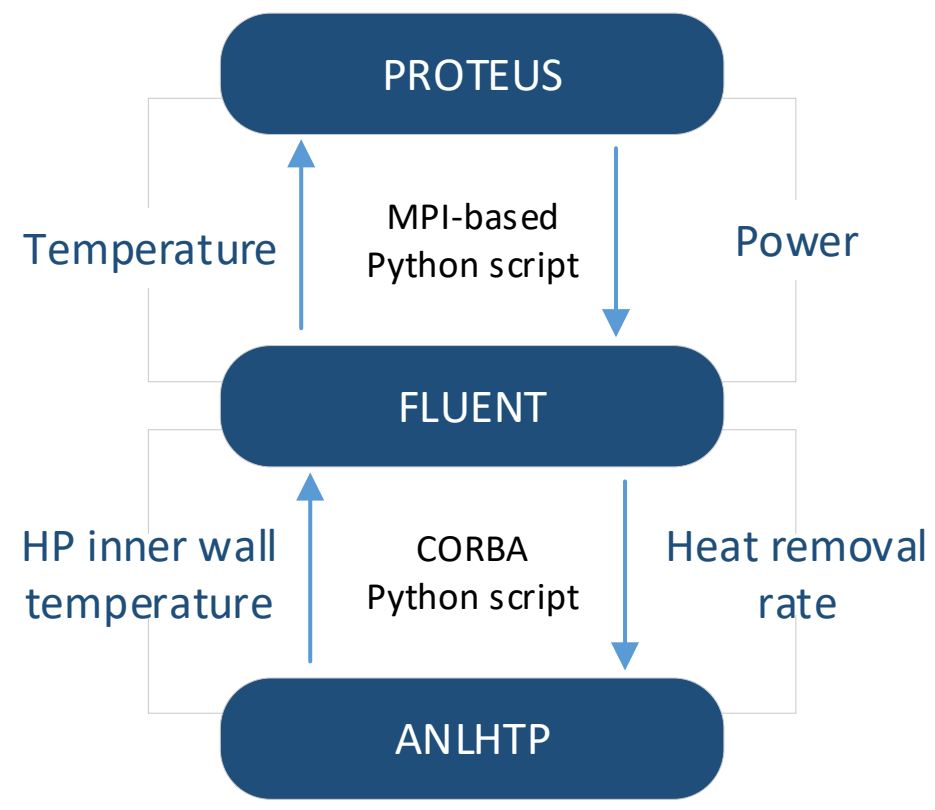

Figure 4-1. Data Exchange of PROTUS, FLUENT, and ANLHTP

In addition, we found that the ANSYS / FLUENT mesh exported in the Exodus format is difficult to be made compatible with PROTEUS because it is complicated to map region names (too many regions to manage) with compositions and identify boundary surfaces for setting up boundary conditions. In this study, therefore, meshes are constructed for each of PROTEUS and FLUENT, transferring data between the two codes based on their own meshes and requiring the 
interpolation from one to the other meshes. FLUENT interpolates the power data transferred from PROTEUS using its built-in interpolation module, whereas the PROTEUS Python driver interpolates the temperature data given from FLUENT to provide the interpolated data to PROTEUS. The MPI-based Python makes it possible to transfer the interpolated data to PROTEUS via a memory instead of a file, once data are read and interpolated by the Python driver.

The coupling of PROTEUS and FLUENT is controlled by two separate Python drivers. The PROTEUS Python driver controls the overall system as well as PROTEUS, while the FLUENT Python driver controls the coupling of FLUENT and ANLHTP. The two Python drivers properly coordinate the simultaneous execution of the three codes to perform the workflow of coupled simulation, running or holding a code for a certain segment of calculations while the other codes generate the data required for the subsequent calculations. The Common Object Request Broker Architecture (CORBA) protocol [24] allows us to control FLUENT externally, executing or holding the code until the input data are updated by ANLHTP (temperatures at the wick-vapor interface of heat pipes) or PROTEUS (power). Figure 4-2 shows the coupling scheme of the three codes controlled by the two Python drivers.

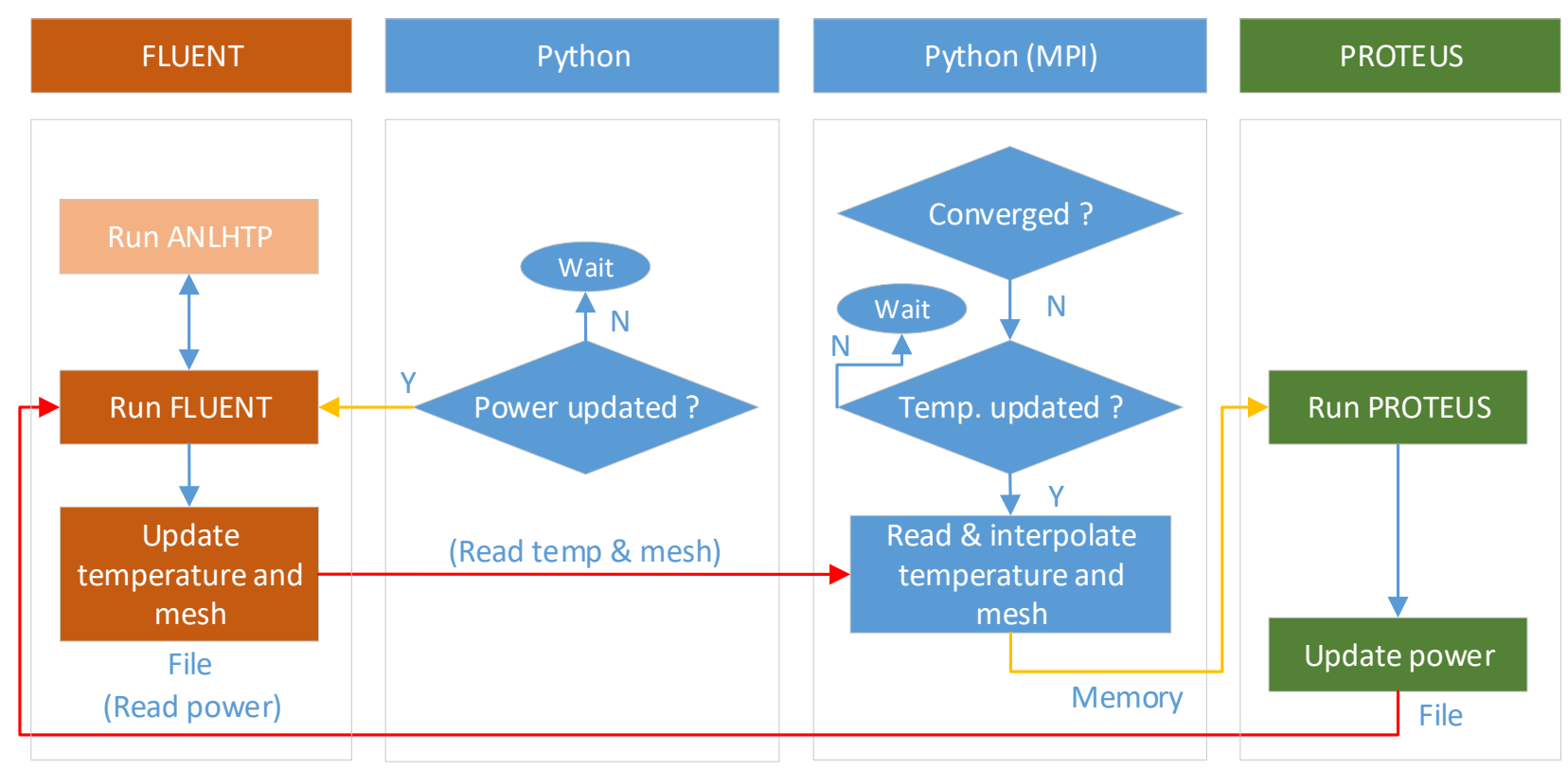

Figure 4-2. Data Exchange of PROTUS, FLUENT, and ANLHTP

In the coupling of FLUENT / ANLHTP, FLUENT is used for the monolith and fuel thermal conduction analysis, while ANLHTP calculates heat pipe temperatures and operation limits. In the process of the coupling, FLUENT and ANLHTP exchange the wick-vapor interface temperature and heat removal rate from heat pipe evaporators to determine the temperature distribution of a core as shown in Figure 4-3. This implies that the FLUENT domain includes the 
wick and heat pipe wall. A 3D transient conduction equation is applied to these regions to consider the non-uniform axial power distribution and thermal inertia of the liquid and wick in the evaporator. The effective thermal conductivity and heat capacity are calculated in FLUENT based on the wick geometry information.

Meanwhile, the regions analyzed by ANLHTP are the vapor core and the condenser, i.e. thermal resistances 4-11 shown in Figure 2-3. ANLHTP is the lumped parameter code unlike FLUENT and therefore, it is assumed that the wick-vapor interface temperature is uniform along the evaporator. Moreover, the thermal inertia of the vapor core is assumed to be negligible since the vapor mass in the core is relatively very small with low pressure inside the heat pipe. This approach can be defined as the quasi-steady-state model in accordance with the Zuo's transient analysis method [25], which showed little difference from a fully transient model.

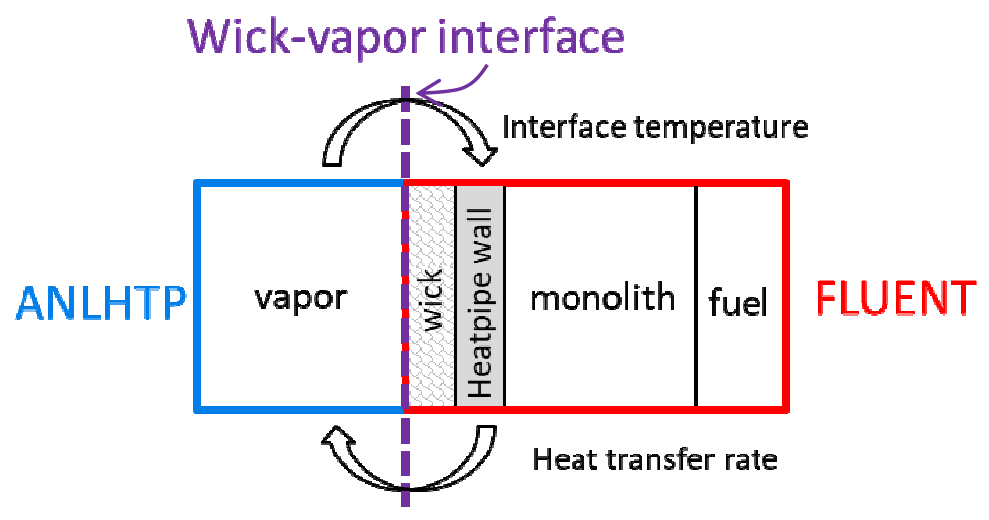

Figure 4-3. Boundary Conditions of the Coupled Simulation of FLUENT and ANLHTP

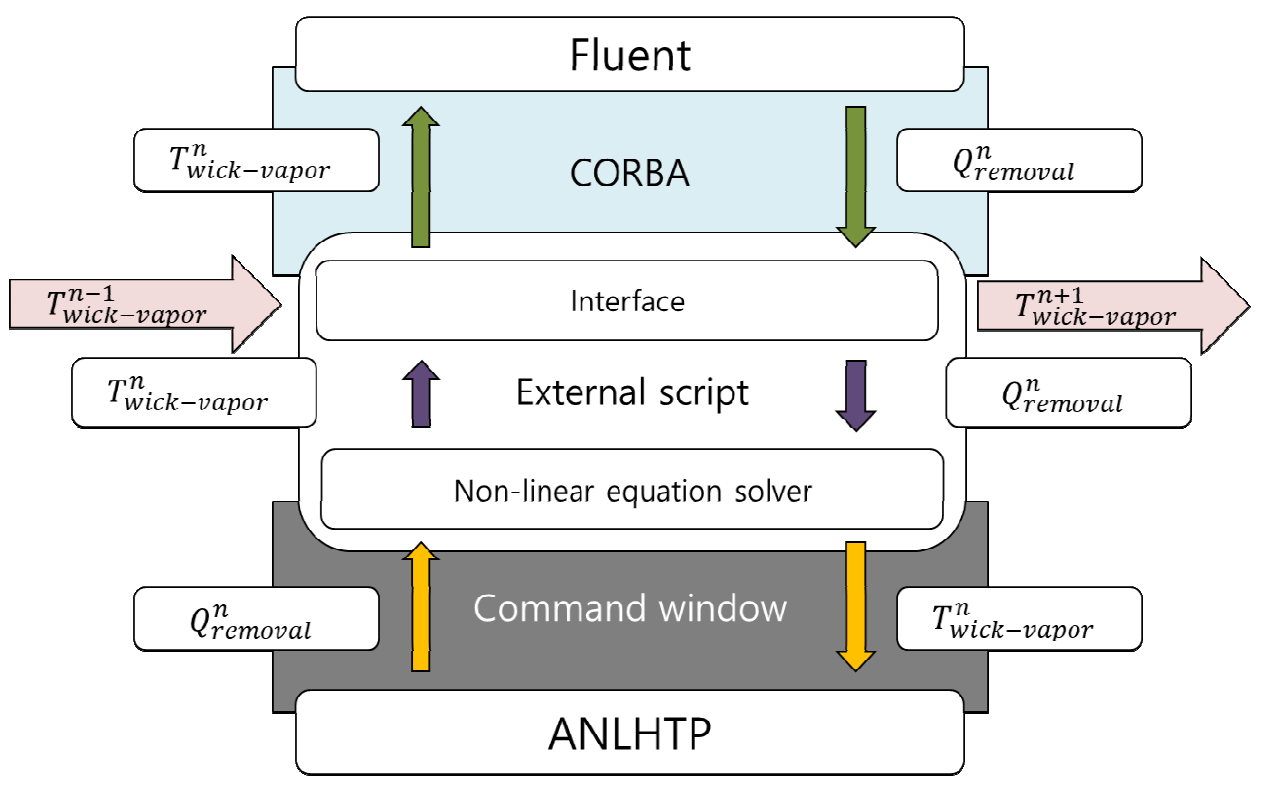

Figure 4-4. Coupled Simulation Procedure for a Single Time Step

The coupling requires data exchange between the two codes which was conducted using the function directed by "ANSYS FLUENT As a Server." This is a set of tools offering the functionality that allows local or remote client applications to access a full capability of the 
FLUENT solver. A client application can perform case setup, initialization, iteration, and result reporting using FLUENT as a Server interface. In the present work, a Python driver was written for the client application, controlling FLUENT using the Common Object Request Broker Architecture (CORBA) protocol [24]. This is a standard protocol defined by the Object Management Group (OMG) designed to facilitate the communication of systems that are deployed on diverse platforms and enables communication between codes written in different languages and running on different computers. Using the protocol, the Python driver controls the boundary conditions of the FLUENT calculation and extract the required data for the coupled calculation at every time step.

Figure 4-4 describes the coupled simulation procedure for a single time step. At the first step of the coupled calculation, initial values are imposed for the wick-vapor interface temperatures. The values are then transferred to FLUENT as boundary conditions by the Python driver. With the boundary conditions, FLUENT calculates the temperature distribution in the fuel, monolith, heat pipe wall and the wick region. The heat flux distribution is estimated based on the calculated temperature gradient on the wick-vapor interface. The calculated heat flux distribution is integrated along the wick-vapor interfaces of each heat pipe to evaluate the heat transfer rates which are transferred to ANLHTP via the Python driver for the interface temperature calculation.

ANLHTP requires the wick-vapor temperature as an input and produces the heat transfer rate as an output. Thus, an iterative procedure was implemented to determine the interface temperature which satisfies the given heat removal rate. Once the solution converges, the determined wick-vapor interface temperature is transferred to FLUENT via the Python driver to repeat the conduction calculation with the updated boundary condition. This procedure is repeated at a single time step until the solution convergence is achieved. The coupling requires two iterative loops within a time step; one for ANLHTP to find the wall temperature satisfying the given wall heat transfer rate and the other to find the converged solution of the Picard iteration between the two codes. This implicit coupling method is advantageous in the transient simulation by allowing a large time step value. In the case of transient analysis, the procedure described above is repeated during the time marching.

\subsection{Coupled Calculation Results}

\subsubsection{Steady-state Single Heat Pipe Problem}

As the first verification of the coupling, a single heat pipe conceptual problem was defined, which was surrounded by monolith and six fuel rods. In this single heat pipe problem, only one iteration step is necessary between FLUENT and ANLHTP as all the heat generated from the fuel rods is supposed to be removed by the heat pipe. Therefore, the objective of this conceptual problem is to check the coupling process and confirm the agreement in the heat pipe outer wall temperatures between the two codes.

The geometry and mesh scheme for this conceptual problem are shown in Figure 4-5. The detail dimensions were obtained from the core configuration of MegaPower. 88,260 elements were used with 30 layers in the axial direction. An axially cosine-shaped power distribution with total 4,680 W was applied for the core region, which was obtained from PROTEUS.

The calculated temperature distribution are shown in Figure 4-6. The result shows that the temperature distribution corresponding to the cosine shaped power distribution was properly calculated. The outer wall temperature calculated from ANLHTP was compared with the area 
averaged temperature along the heat pipe outer wall in FLUENT. The resulting ANLHTP and FLUENT temperatures were almost the same as each other, $906.55 \mathrm{~K}$ and $906.56 \mathrm{~K}$, respectively, indicating that the energy conservation between the two codes was almost satisfied.

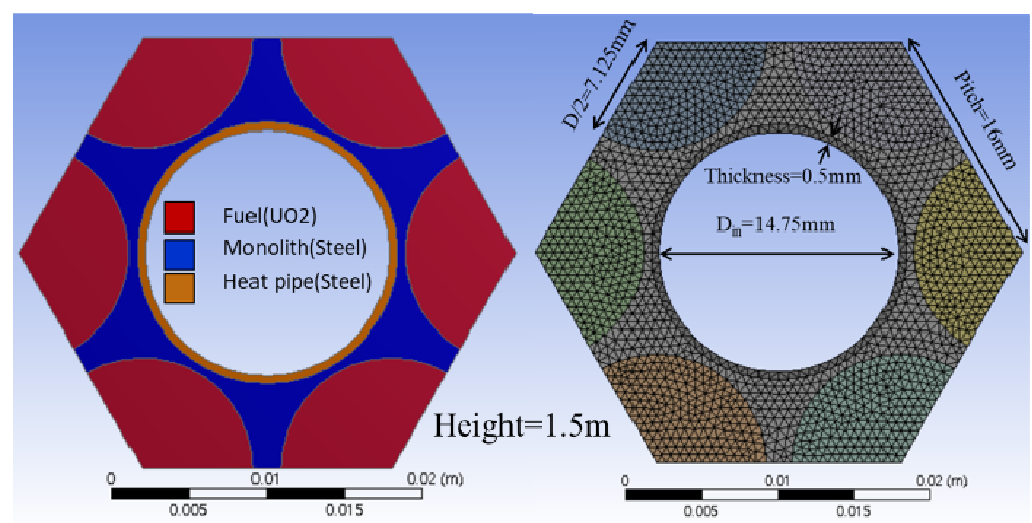

Figure 4-5. Geometry and Mesh Scheme of Single Heat Pipe Problem

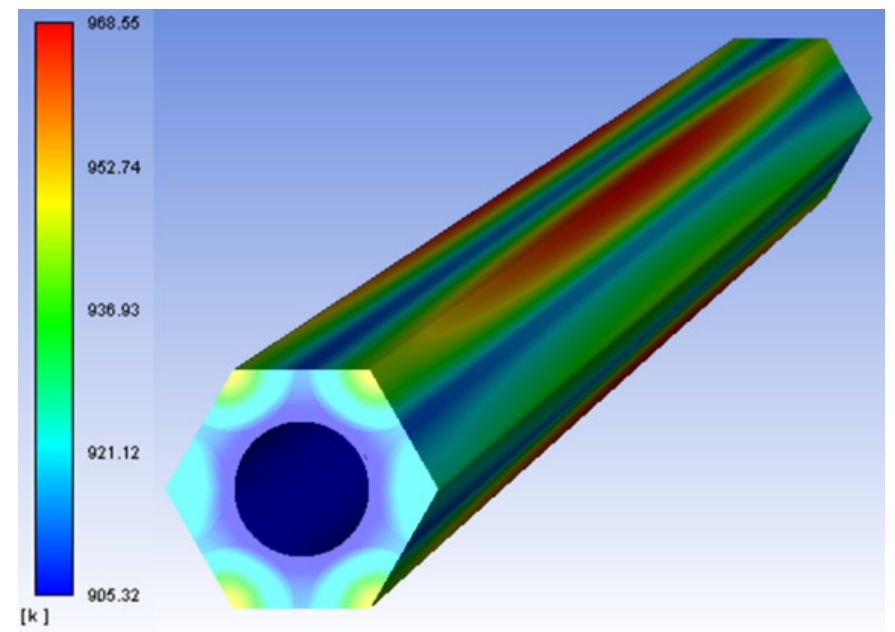

Figure 4-6. Temperature Distribution for Single Heat Pipe Problem

\subsubsection{Steady-state Multi Heat Pipe Problem}

A heat pipe conceptual unit assembly problem was developed based on the core configuration of MegaPower was analyzed to verify the coupled system. In this conceptual problem, six fuel rods were surrounded by seven heat pipes in the monolith. The powers for six fuel rods were given as shown in Table 4-1, and the mesh and geometry used in the calculation are shown in Figure 4-7. The 3D problem was divided into 30 axial layers. The given total power was 14,400 $\mathrm{W}$, and $\sim 14 \%$ lower power with an axially cosine shaped profile was applied to the fuel rods 2 and 5 in order to generate asymmetric temperature distributions. Two different meshes (71,680 and 171,720 elements) were tested to confirm mesh convergence. The heat pipe inner wall temperatures obtained with the two meshes showed a difference of less than $0.1 \mathrm{~K}$. Therefore, the coarser mesh was selected for the calculation. 


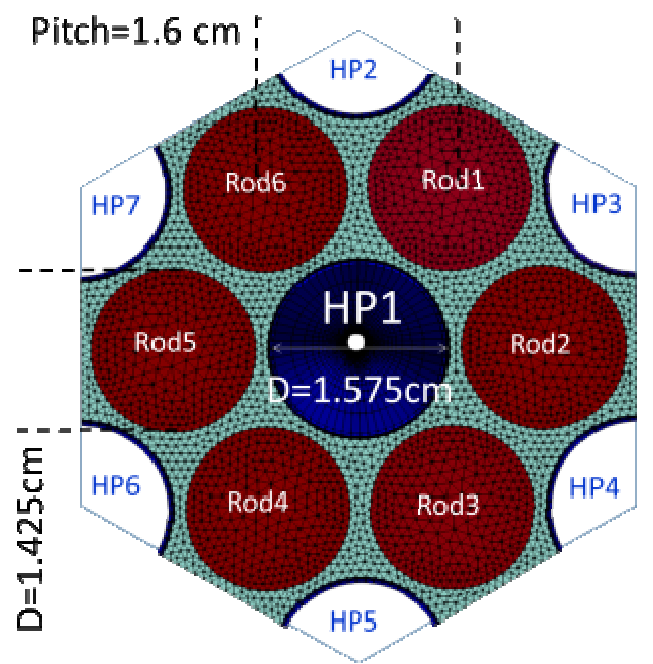

Figure 4-7. Geometry and Mesh Scheme of Multi-Heat Pipe Problem

Table 4-1. Fuel Rod Powers for FLUENT / ANLHTP with the Steady-state Condition

\begin{tabular}{|c|c|}
\hline Rod number & Power $(\mathrm{W})$ \\
\hline 1 & 2,520 \\
2 & 2,160 \\
3 & 2,520 \\
4 & 2,520 \\
5 & 2,160 \\
6 & 2,520 \\
\hline
\end{tabular}

The calculated temperature distribution and convergence of the heat pipe inner wall temperatures are shown in Figure 4-8 and Figure 4-9, respectively. The higher temperature values in the mid-plane were caused by the cosine shaped axial power distribution. The heat pipe temperatures were determined mostly by the powers generated from neighboring fuel rods. Based on given powers of the fuel rods, the inner wall temperatures of heat pipes 2 and 5 should be the highest, that of heat pipe 1 which is located at the center is to be the second highest, and those of heat pipes 3, 4, 6 and 7 should be the same lowest. Figure 4-9 shows apparently that the heat pipe temperatures converged to reasonable values, indicating that the coupled codes performed well for the steady-state calculation. 


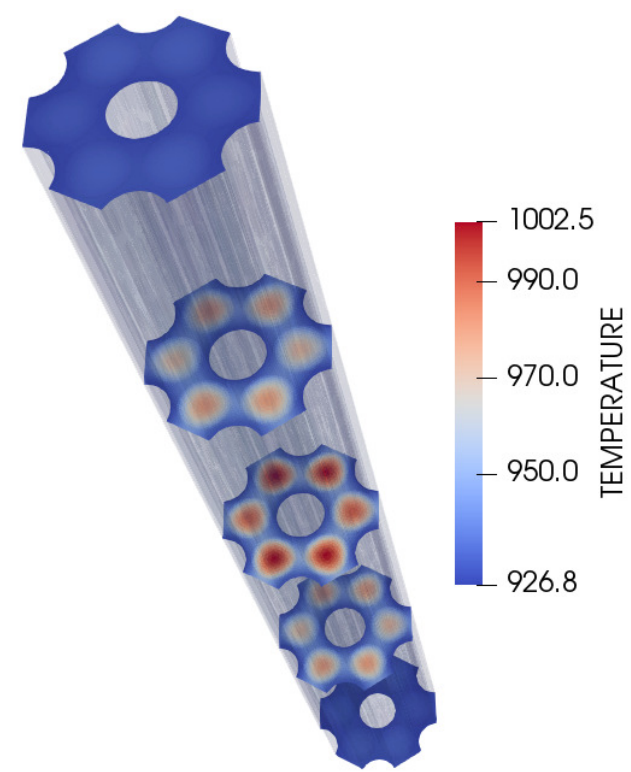

(Scale ratio $\mathrm{x}: \mathrm{y}: \mathrm{z}=1: 1: 0.25)$

Figure 4-8. Temperature Distribution of Multi-Heat Pipe Problem

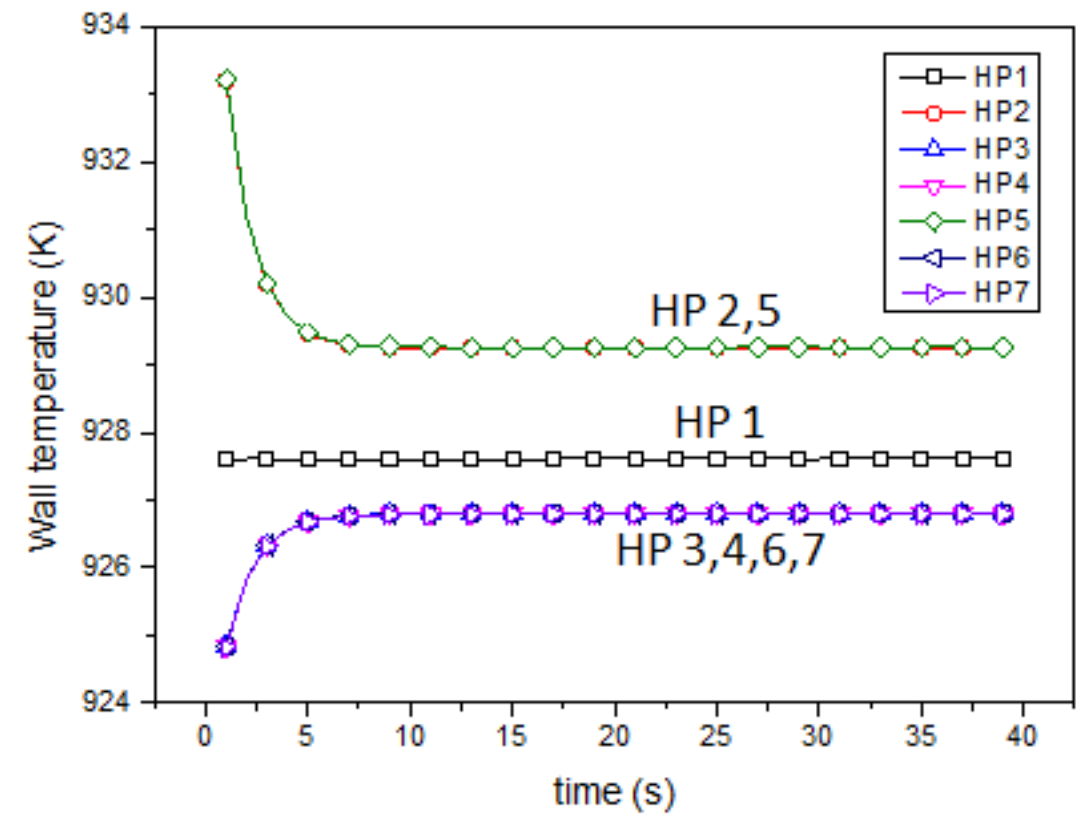

Figure 4-9. Heat Pipe Wall Temperature Convergence Result of Multi-Heat Pipe Problem

\subsubsection{Transient Multi Heat Pipe Problem}

The transient performance of the coupled codes was demonstrated using the 3D unit fuel assembly problem. As a postulated event of the micro reactor core, failure conditions of a heat pipe in the unit assembly were analyzed. The transient of the heat pipe failure was simulated in the following two steps: at first, it was analyzed using FLUENT / ANLHTP without accounting 
for neutronics feedback and then, the same condition was analyzed again using PROTEUS / FLUENT / ANLHTP in order to consider the neutronics feedback.

\section{Transient analysis without neutronics feedback}

Initially, a steady-state calculation was conducted to find out a normal operational condition. Different from the previous case where powers are not symmetric, the symmetric power profile generated from PROTEUS was applied. The total power imposed at the steady-state condition was $13,968 \mathrm{~W}$ for this case. Then, transients with a single heat pipe failure were initiated from the steady-state temperature distribution. It was assumed that when a heat pipe failed, the boundary condition for the wick-vapor interface was turned to be the adiabatic wall immediately. Note that the power distribution used in the steady-state calculation remained the same during the entire transient time period: i.e., no power feedback was accounted for this test.

Two different cases of the single heat pipe failure problem were defined: heat pipe 1 (HP1) failure problem (Case-1) and HP2 failure problem (Case-2). The locations of the heat pipes are indicated in Figure 4-7. Figure 4-10 and Figure 4-11 present the resulting temperature distributions at the end of transient and the wick-vapor interface temperatures with time for Case1, respectively. In Case-1, the failure of HP1 at the center of the assembly led to the increase of the overall temperature due to the absence of cooling source. As the monolith temperature increased, more heat was removed by the operating heat pipes, as shown in Figure 4-11. The temperatures of the intact heat pipes were evenly increased because of the symmetric locations of the intact heat pipes. The calculation was terminated at $500 \mathrm{sec}$ when the temperatures reached another stable conditions.

In Case-2, one of the surrounding heat pipes, HP2, was made fail. Figure 4-12 and Figure 4-13 show the results of temperature distributions and wick-vapor interface temperatures in the Case- 2 simulation. As expected, a strongly asymmetric temperature distribution appeared and the maximum temperature was shifted toward the location of HP2. The maximum temperature was relatively lower than that in Case-1 because the heat removal rate of HP2 was one-third of HP1. As a result, the temperature increase of the other heat pipes in Case- 2 showed less than that in Case-1 as well. HP3 and HP7 showed the largest temperature increase, as they were the closest to the failed heat pipe. HP1 has the same distance from HP2 but its surface area was three times larger than the others in the unit assembly. Thus, the temperature increase of HP1 was smaller than HP3 and HP7. The other heat pipes also showed temperature increases smaller than HP1 because they were at the farthest locations from the failed heat pipe in the assembly.

\section{Transient analysis with neutronics feedback}

In this transient simulation, the power feedback to FLUENT was accounted for using PROTEUS / FLUENT / ANLHTP. First of all, a steady-state calculation was performed using the three codes. The thermal feedback effect was accounted for in the power calculation of PROTEUS, and the power feedback was considered in the temperature calculation of FLUENT. When the steady-state solution was converged, the transient calculation was started by initiating the HP2 failure immediately. As shown in the FLUENT / ANLHTP calculation above, the failure of HP2 led to the increase of the temperature at HP2 until 170 sec which was propagated to the neighboring regions. However, due to the Doppler feedback, the power decreased as the fuel temperatures increased, leading to the reduction of the temperature increase rate and finally the decrease of HP temperatures starting from $\sim 170 \mathrm{sec}$. Since the power kept decreasing, the HP temperatures decreased up to below the temperatures at the steady-state condition. As 
temperatures decreased below the steady-state condition, the decrease rate of power was reduced due to the negative Doppler reactivity and consequently the decrease rate of temperatures was slowed down as well. A negative reactivity feedback was initiated due to the temperature increase at the HP2 failure, whose magnitude was reduced due to the temperature decrease. As the negative reactivity was reduced to almost zero, the power and temperature of the benchmark problem were converged to another stable condition in terms of temperature and power.

Figure 4-14 shows the power and temperature changes with time after the transient started. The final temperature of HP2 became higher than the initial before the transient started and the temperatures of the other heat pipes were lower than those at the initial steady-state condition, as illustrated in Figure 4-15. As seen in the previous transient case without neutronics feedback, the temperature of HP3 and HP7 were the second highest, that of HP1 was next, and those of HP4 and HP6 were the same next, and that of HP5 was the lowest which was the farthest from the fail heat pipe. The transient results from the coupled simulation of PROTEUS / FLUENT / ANLHTP appear to be reasonable qualitatively, indicating that the coupled system were implemented correctly. Further verification tests will be conducted for different transient cases as well as larger or whole-core benchmark problems. Test results will be verified quantitatively as well. 


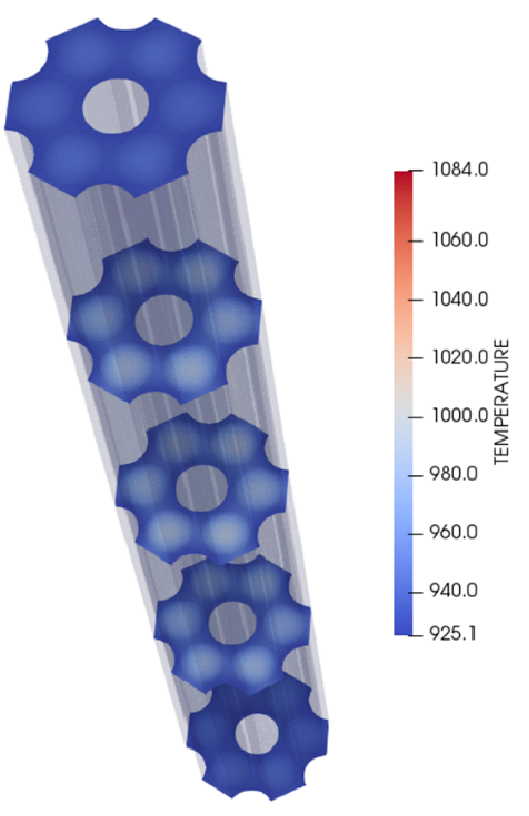

(a) Before transient

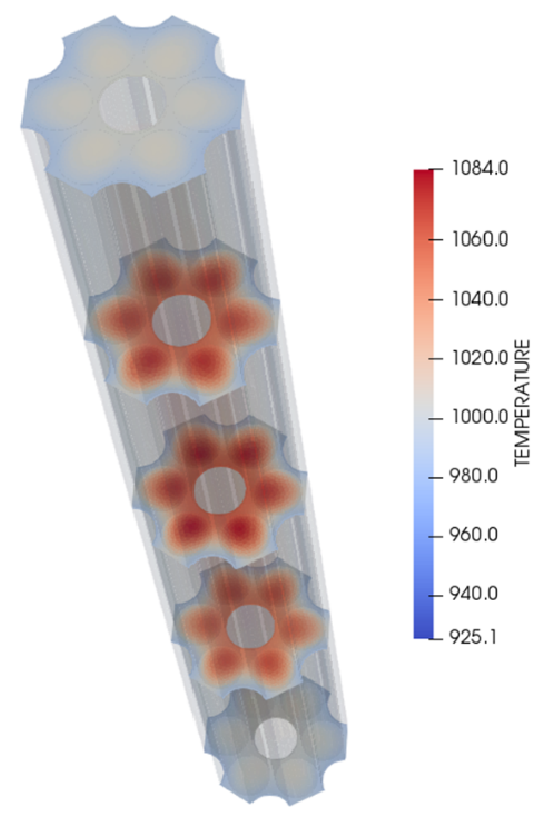

(b) After transient

Figure 4-10. Temperature Distributions of the HP1 Failure Problem Before and After Transient

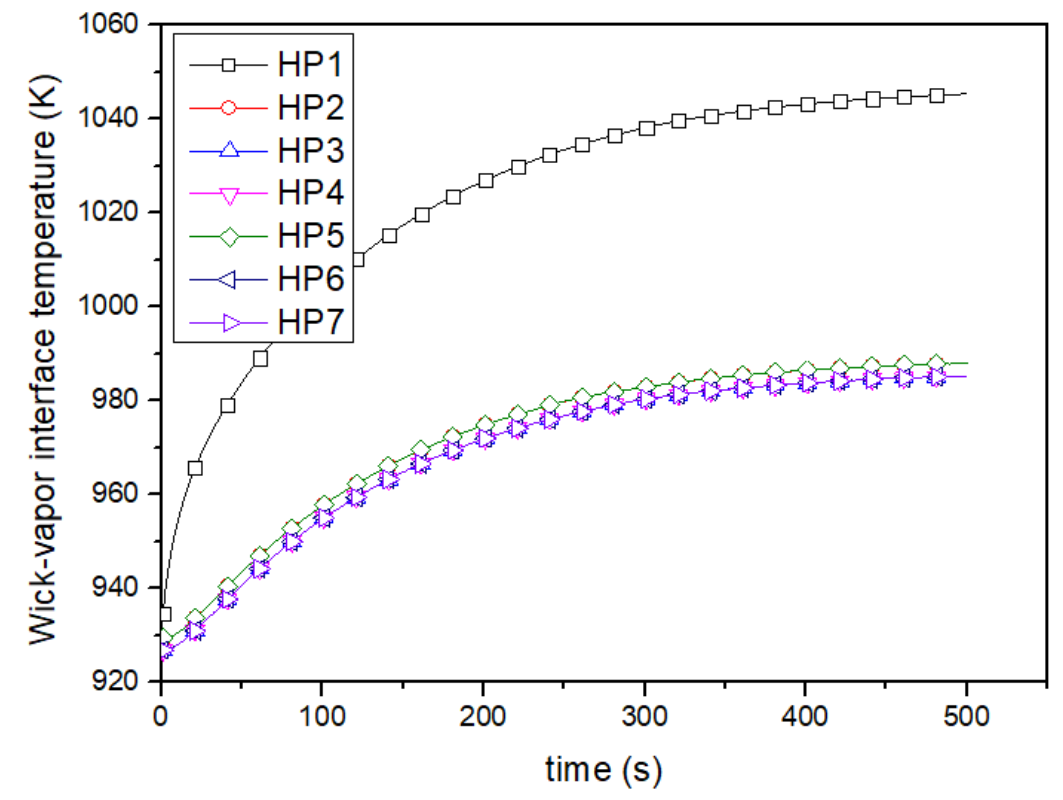

Figure 4-11. Wick-vapor Interface Temperature Transient for the HP1 Failure Problem 


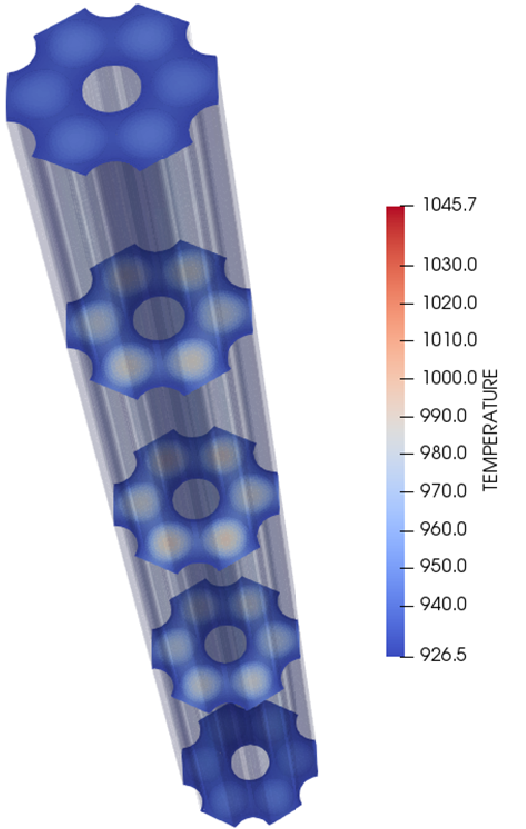

(a) Before transient

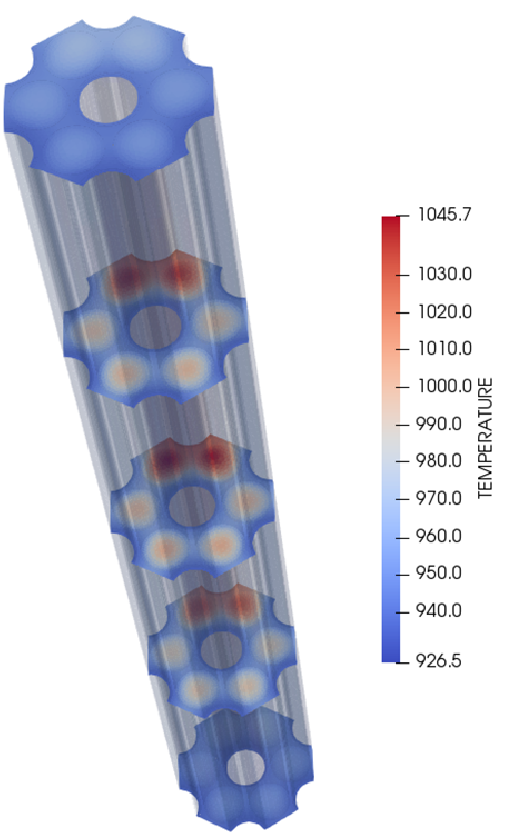

(b) After transient

Figure 4-12. Temperature Distributions of the HP2 Failure Problem Before and After Transient

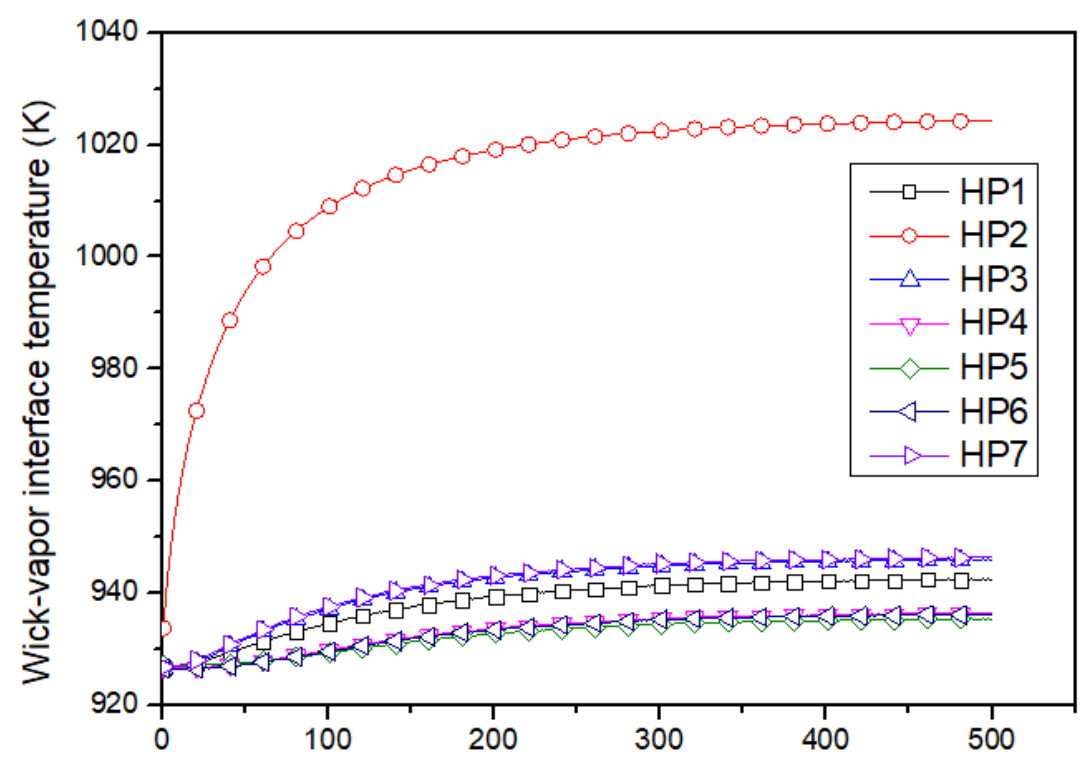

Figure 4-13. Wick-vapor Interface Temperature Transient for the HP2 Failure Problem 

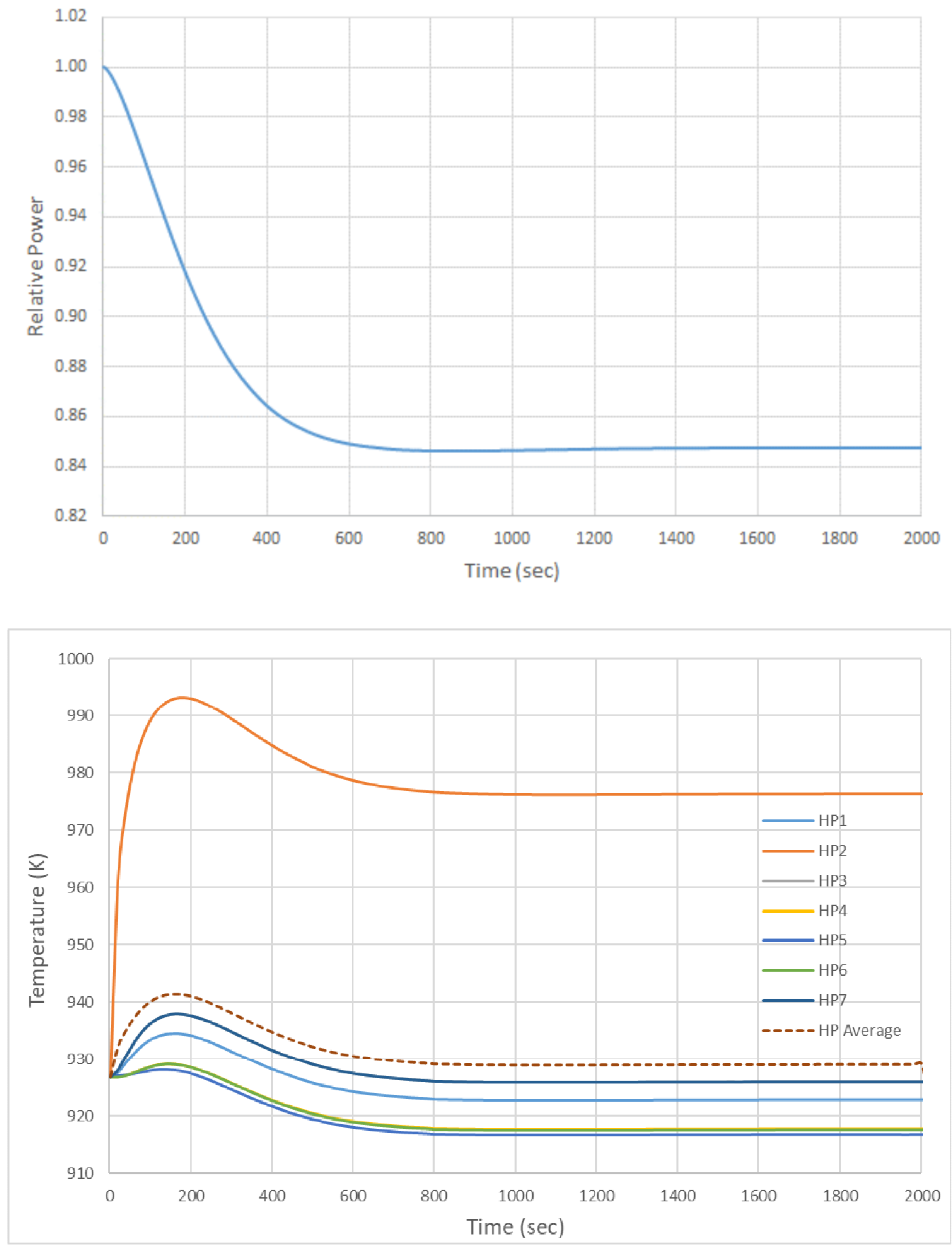

Figure 4-14. Relative Power (Top) and Heat Pipe Temperature (Bottom) Change with Time for One Heat Pipe Failure Transient Problem 

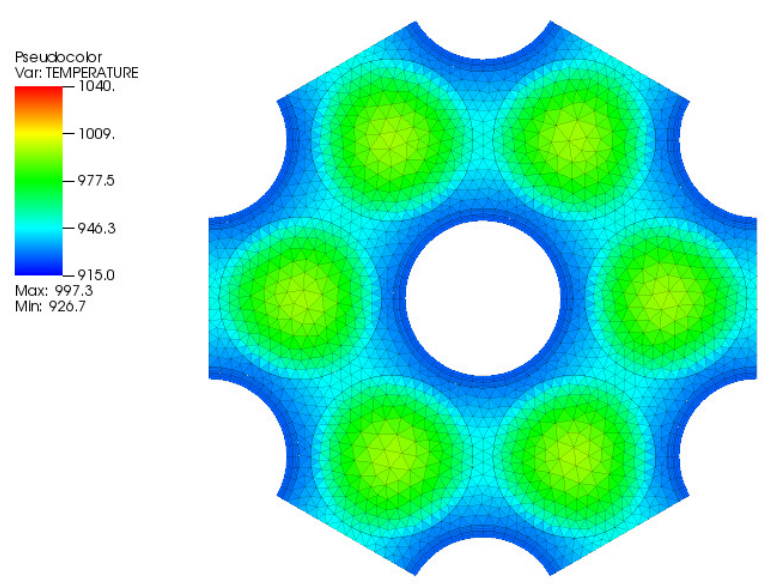

$\mathrm{t}=0 \mathrm{sec}$
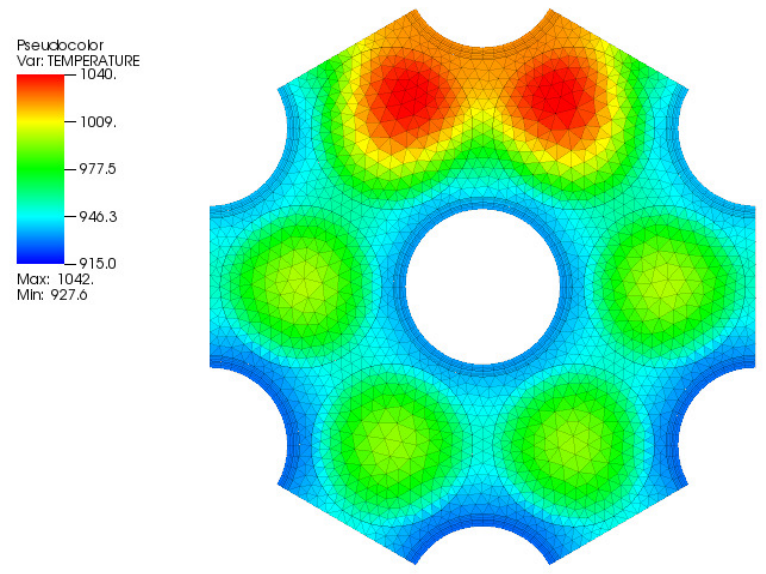

$\mathrm{t}=200 \mathrm{sec}$
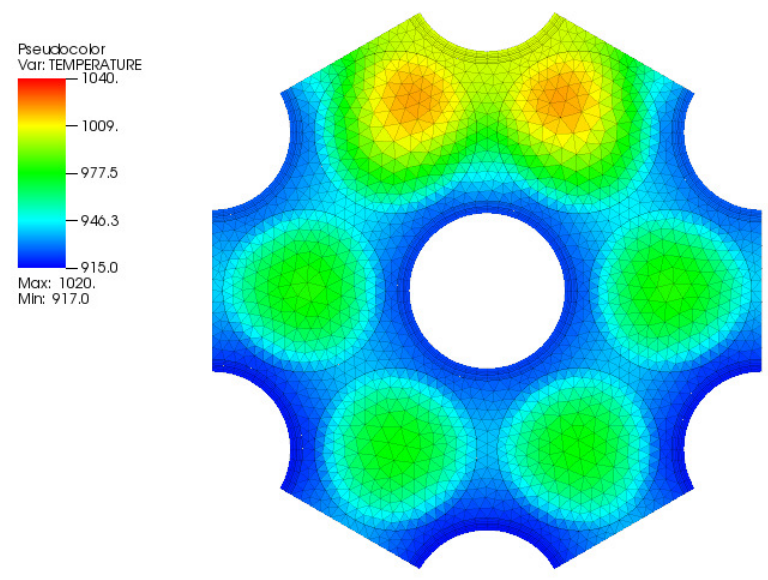

$$
\mathrm{t}=800 \mathrm{sec}
$$

Figure 4-15. Temperature Distributions with Time for the Transient Case with One Heat Pipe Failure

\section{Conclusions}

Two micro reactor cores were simulated using the MOC solver of PROTEUS: MegaPower for a heat pipe micro reactor and Holos-Quad for a high-temperature gas-cooled micro reactor. For 
verification of PROTEUS neutronics solutions, geometry and meshes for PROTEUS were generated using CUBIT with the support of the Argonne mesh toolkit, cross sections were generated using SERPENT / GenISOTXS, and PROTEUS solutions were compared with SERPENT Monte Carlo solutions. Multiphysics simulations were also performed using the coupled system of PROTEUS / FLUENT / ANLHTP, which was applied to the heat pipe cooled micro reactor benchmark problems.

For MegaPower, benchmark problems including a fuel pin, a unit cell, a core with fuel only, and $2 \mathrm{D}$ and $3 \mathrm{D}$ cores with reflector and shield regions were developed. 17- and 33-group multigroup cross sections were produced using SERPENT / GenISOTXS. Since MegaPower is a fast-spectrum core, an additional cross section set was generated using $\mathrm{MC}^{2}-3$ as well. With the SERPENT cross sections, the eigenvalue comparison between PROTEUS and SERPENT showed overall good agreement within $250 \mathrm{pcm}$, except for the case with control material in the control drums turned to the nearest locations of the core. However, with the $\mathrm{MC}^{2}-3$ cross sections, the PROTEUS eigenvalues showed better agreement with the SERPENT solutions within $144 \mathrm{pcm}$ for all core cases. The large difference in the eigenvalue with the SERPENT cross sections would be attributed to inaccurate high-order scattering moments since SERPENT calculates high-order scattering moments using the scalar flux weighting instead of the moment weighting. Further investigation and verification will be performed in future.

For Holos-Quad, a unit cell and 2D and 3D cores were developed with different core configurations in terms of subcritical power module (SPM) locations, hafnium control blade, fuel and coolant temperatures. 14-group cross sections were generated using SERPENT. The PROTEUS eigenvalues for the 2D and 3D cores were in good agreement with SERPENT within 101 and 227 pcm, respectively. The MDC and DTC of reactivity were in good agreement between the two codes within $9 \%$ as well.

For a heat pipe cooled micro reactor, multiphysics simulations were performed using PROTEUS for neutronics analysis, FLUENT for thermal analysis, and ANLHTP for heat pipe performance analysis. Note that FLUENT was used temporarily in the study, instead of ANSYSmechanical, because of the limited code availability. The coupling system of the three codes were developed using the Python-based external drivers, which coordinate the overall workflow including data exchange (power, temperature, and heat rate) required for the coupling and also control the individual calculation steps of the three codes such as convergence check and boundary conditions. The coupled system was qualitatively verified using a conceptual 3D unit assembly problem composed of six fuel rods and seven heat pipes, which was developed based on the compositions of MegaPower.

First, the steady-state problem was solved by the coupled system of FLUENT / ANLHTP only with given asymmetric fuel powers, demonstrating the reasonable convergence of temperatures of 7 heat pipes in hexagonal lattice configuration. In addition, transient simulations were also performed by making one out of 7 heat pipes (a heat pipe at the center or outside) fail. In particular, the coupled system of PROTEUS / FLUENT / ANLHTP were tested for the case of one heat pipe failure (failing one of the surrounding six heat pipes), showing reasonable changes of total power and heat pipe temperatures with time accounting for temperature feedback effects. Since ANLHTP was a steady-state code, it was slightly updated for slow transient simulation, assuming that the vapor core has relatively negligible thermal inertia and a quick response with the change of the wick-vapor interface temperature. This assumption allows the code to analyze the vapor core using the steady-state model. Note that the components of a heat pipe (heat pipe 
container, wick and liquid in the wick) that have relatively large thermal inertia were excluded from ANLHTP and were modelled using FLUENT. Meanwhile, no effect of structural change was accounted for in the study, which will be performed using ANSYS-mechanical in future with further verification tests using larger or whole-core $3 \mathrm{D}$ problems. 


\section{REFERENCES}

1. P. R. Mcclure, D. I. Poston, V. R. Dasari, and R. S. Reid, "Design of Megawatt Power Level Heat Pipe Reactors," LA-UR-15-28840, Los Alamos National Laboratory (2015).

2. J. W. Sterbentz et al., "Reactor (5 MW) for Reliable Power at Remote Sites Assessment Report Using Phenomena Identification and Ranking Tables (PIRTs)," INL/EXT-16-40741, Rev.1, Idaho National Laboratory (2017).

3. A. Levinsky, J. J. V. Wyk, Y. Arafat, and M. C. Smith, "Westinghouse eVinci Reactor for off-Grid Markets," ANS Winter meeting, Orlando, Florida, November 11-15 (2018).

4. N. E. Stauff, C. H. Lee, P. Shriwise, Y. Miiao, R. Hu, P. Vegendla, and T. Fei, "Neutronic Design and Analysis of the Holos-Quad Concept," ANL/NSE-19/8, Argonne National Laboratory, June (2019).

5. E. R. Shemon, M. A. Smith, and C. H. Lee, "PROTEUS-SN Methodology Manual," ANL/NE-14/5, Argonne National Laboratory, June (2014).

6. Y. S. Jung, C. H. Lee, and M. A. Smith, "PROTEUS-MOC User Manual," ANL/NE-18/10 Rev. 0, Argonne National Laboratory, September (2018).

7. Y. S. Jung, C. H. Lee, and M. A. Smith, "PROTEUS-NODAL User Manual," ANL/NE-18/4 Rev. 0, Argonne National Laboratory, September (2018).

8. CUBIT Web page, www.cubit.sandia.gov.

9. M. A. Smith and E. R. Shemon, "User Manual for the PROTEUS Mesh Tools," ANL/NE15/17 Rev. 2, Argonne National Laboratory, September (2016).

10. J. LEPPANEN, "Serpent - a Continuous-energy Monte Carlo Reactor Physics Burnup Calculation Code," VTT Technical Research Centre of Finland, June (2015).

11. N. E. Staff, P. K. Romano, C. H. Lee, and T. K. Kim, "Verification of Mixed Stochastic/Deterministic Approach for Fast and Thermal Reactor Analysis," ICAPP 2017, Fukui and Kyoto, Japan, April 24-27 (2017).

12. C. H. Lee and W. S. Yang, "MC'-3: Multigroup Cross Section Generation Code for Fast Reactor Analysis," ANL/NE-11-41 Rev. 3, Argonne National Laboratory (2018).

13. ANSYS Web page, www.ansys.com.

14. G. A. McLennan, "ANL/HTP: A Computer Code for the Simulation of Heat Pipe Operation," ANL-83-108, Argonne National Laboratory (1983).

15. FLUENT Web page, www.ansys.com/products/fluids/ansys-fluent.

16. M. A. Smith, E. E. Lewis, and E. R. Shemon, "DIF3D-VARIANT 11.0: A Decade of Updates," ANL/NE-14/1, Argonne National Laboratory, January (2014).

17. M. H. BAE, "Development of Triangle-based Polynomial Expansion Nodal $\mathrm{SP}_{3}$ Method for Hexagonal Core Transport Calculation," Seoul National University (2010).

18. S. W. Chi, Heat Pipe Theory and Practice: A Sourcebook, McGraw Hill Book Company, New York (1976).

19. P. Dunn and D. A. Reay, Heat Pipes, Pergamon Press, Oxford (1978).

20. J. E. Kemme et al., "Performance Investigations of Liquid-Metal Heat Pipes for Space and Terrestrial Applications," Proc. 3rd Int. Heat Pipe Conf., Palo Alto, CA, May (1978).

21. R. E. Holtz et al., "On the Experimental Operation of a Sodium Heat Pipe," ANL-85-61, Argonne National Laboratory (1985).

22. C. H. Lee and Y. S. Jung, "Generation of the Cross Section Library for PROTEUS," ANL/NE-18/2, Argonne National Laboratory, January (2018). 
23. P. K. Romano et al., "OpenMC: A State-of-the-Art Monte Carlo Code for Research and Development," Ann. Nucl. Energy, 82, 90 (2015).

24. CORBA Web page, www.corba.com.

25. Z. J. Zuo, A. Faghri, and L. Langston, "Numerical analysis of Heat Pipe Turbine Vane Cooling," J. Eng. Gas Turbines Power 120(4), 735-743 (1998). 


\section{Argonne}

Nuclear Science and Engineering Division

Argonne National Laboratory

9700 South Cass Avenue, Bldg. 208

Argonne, IL 60439-4842

www.anl.gov 